\title{
Unraveling the transcriptional networks that drive oligodendrocyte fate specification in Sonic hedgehog-responsive neocortical progenitors
}

Caitlin C. Winkler ${ }^{1,2,3^{*}}$, Luuli N. Tran ${ }^{4}$, Ellyn P. Milan ${ }^{3}$, Fernando García-Moreno ${ }^{5,6}$, Santos J. Franco ${ }^{3^{*}}$

${ }^{1}$ Department of Biochemistry and Molecular Genetics, University of Colorado School of Medicine, Anschutz Medical Campus, Aurora, CO 80045, USA. ${ }^{2}$ RNA Biosciences Initiative, University of Colorado School of Medicine, Anschutz Medical Campus, Aurora, CO 80045, USA. ${ }^{3}$ Department of Pediatrics, Section of Developmental Biology, University of Colorado School of Medicine, Anschutz Medical Campus, Aurora, CO 80045, USA. ${ }^{4}$ Graduate Program in Molecular Biology, University of Colorado Graduate School - Anschutz Medical Campus, Aurora, CO 80045, USA. ${ }^{5}$ Achucarro Basque Center for Neuroscience, Edificio Sede del Parque Científico de la UPV/EHU, Leioa, Spain. ${ }^{6}$ Ikerbasque - Basque Foundation for Science, María Díaz de Haro, Bilbao, Spain.

*Correspondence: C.C.W. (Caitlin.Winkler@CUAnschutz.edu), S.J.F. (Santos.Franco@CUAnschutz.edu)

In the developing nervous system, progenitors first generate neurons before making astrocytes and oligodendrocytes. We previously showed that increased Sonic hedgehog (Shh) signaling in dorsal forebrain progenitors is important for their production of oligodendrocytes as neurogenesis winds down. Here, we analyzed single-cell RNA sequencing datasets to better understand how Shh controls this neuron-tooligodendrocyte switch in the neocortex. We first identified Shh-responding progenitors using a dataset in which Shh was overexpressed in the mouse dorsal forebrain. Pseudotime trajectory inferences revealed a subpopulation committed to the oligodendrocyte precursor cell (OPC) lineage. Genes upregulated along this lineage defined a pre-OPC state, as cells transitioned from progenitors to OPCs. Using several datasets from wild-type mouse and human embryos at different ages, we confirmed a pre-OPC state preceding OPC emergence during normal development. Finally, we show that pre-OPCs are enriched for a gene regulatory network involving the transcription factor Ascl1. Genetic lineage-tracing demonstrated Ascl1 ${ }^{+}$dorsal progenitors primarily make oligodendrocytes. We propose a model in which Shh shifts the balance between opposing transcriptional networks toward an Ascl1 lineage, thereby facilitating the switch between neurogenesis and oligodendrogenesis.

\section{INTRODUCTION}

Proper assembly of neural circuits in the developing brain requires spatially and temporally coordinated production of many different neuronal and glial cell types. In the developing dorsal forebrain, neural progenitor cells produce excitatory neurons, olfactory bulb interneurons, astrocytes and oligodendrocytes. Both internal and external factors are important for setting up this diversity, by translating spatial and temporal signaling cues into transcriptional outputs that determine cellular identity and function. A major goal in the field is to understand the molecular mechanisms that specify these different cell fates in the proper numbers and with appropriate timing.
The morphogen signaling molecule, Sonic hedgehog (Shh), is important for specifying oligodendrocyte fates in many different regions of the CNS. Studies from our lab previously showed that Shh signaling is both necessary and sufficient for the generation of neocortical oligodendrocytes from dorsal forebrain progenitors (Winkler et al., 2018). We proposed a model for how the timing and location of Shh signals affect the neuron-glia switch during neocortical development. In the early dorsal forebrain, Shh signaling is maintained at low levels to allow normal dorsal-ventral patterning. During this time, dorsal progenitors undergo neurogenesis to make neocortical excitatory neurons. At later stages, Shh ligand reaches the dorsal forebrain via infiltrating 
interneurons and circulating cerebral spinal fluid (Winkler et al., 2018). We showed that this increase in dorsal Shh is critical for instructing progenitors to begin oligodendrogenesis (Winkler et al., 2018) and to produce the full complement of oligodendrocyte lineage cells for the mature brain (Winkler and Franco, 2019). However, the intrinsic pathways that specify an oligodendrocyte fate downstream of Shh remain largely unknown.

In this study, we analyzed several publicly available single-cell RNA sequencing (scRNA-seq) datasets to investigate how Shh signaling influences progenitor cell fate decisions in the developing dorsal forebrain. We first analyzed data from a recent study that overexpressed Shh ligand during mid-corticogenesis in mouse embryos (Zhang et al., 2020). We identified the progenitors whose transcriptomes were enriched for components of the Shh pathway. We uncovered further diversity within these Shh-responding progenitors, with 2 main lineage trajectories predicted to generate either oligodendrocyte precursor cells (OPCs) or olfactory bulb interneurons (OB-INs). Within the OPC lineage, we identified a set of genes that defines a pre-OPC state between radial glial cells and OPCs. Using this gene set, we identified preOPCs in several other scRNA-seq datasets collected from unmanipulated wild-type mouse embryos at different stages of corticogenesis. We also identified a pre-OPC progenitor state in data from human fetal neocortex and found that human pre-OPCs share some common gene expression profiles with outer radial glial cells. Finally, we identified the transcriptional regulator, Ascl1, as a central component of a gene regulatory network enriched in pre-OPCs. We confirmed that Ascl 1 mRNA and protein are expressed in a subset of dorsal forebrain progenitors prior to OPC production, and demonstrate that $\mathrm{Ascl}^{+}$progenitors primarily generate oligodendrocytes at late embryonic ages. These data support a model of how increased Shh levels during late corticogenesis are translated by dorsal progenitors into a switch in cell fates between neurons and oligodendrocytes.

\section{RESULTS}

\section{Identification of Shh-responding progenitor cells}

To gain insights into how Shh signaling promotes oligodendrogenesis in the dorsal forebrain, we analyzed published scRNA-seq data in which Shh was ectopically expressed in the cortical ventricular zone (VZ) via in utero electroporation (IUE) at E13.5 (Zhang et al., 2020). Samples were collected at E16.5 for scRNA-sequencing analysis (Fig. 1A). This gave us a system to study the molecular pathways downstream of Shh signaling in dorsal forebrain neural progenitors. We first identified major cell types based on gene expression (Fig. 1, Table $\mathrm{S}_{1}$ ). As expected, most cells were excitatory projection neurons at various stages of development (Fig. 1A-B). Progenitor cells, which included radial glial cells (RGCs), intermediate progenitor cells (IPCs), and oligodendrocyte precursor cells (OPCs), represented 4 clusters (Fig. 1A-C). We also identified 2 distinct inhibitory interneuron (IN) clusters (Fig. 1A, C), one of which comprised INs generated in the ventral forebrain that had migrated into the neocortex. The other IN cluster was classified as precursors for olfactory bulb interneurons (OB-INs), based on differential expression of OB-IN markers.

We then re-clustered the progenitor cells for more detailed analysis. Because of their similarities to the progenitor clusters, we also included the immature OB-INs in our further analysis. Reclustering revealed 9 progenitor clusters, representing 5 different major cell types (Fig. 2A). The majority (56\%) of cells were IPCs (Eomes ${ }^{+}$) at various stages of development toward excitatory neurons (Fig. 2A, B, D). RGCs $\left(F a b p 7^{+}\right)$constituted $15.5 \%$ of all progenitor cells (Fig. $2 \mathrm{~A}, \mathrm{~B}, \mathrm{C})$. OB-INs $\left(S p 8^{+}\right)$divided into two clusters, likely based on maturation state, and constituted $12.8 \%$ of all progenitors (Fig. 2A, B, F). OPCs $\left(\mathrm{Olig}_{1}{ }^{+}\right)$ made up $2.8 \%$ of progenitors (Fig. $2 \mathrm{~A}, \mathrm{E}$ ). The final progenitor cluster did not fall into any of these classifications (Fig. 2A-F). This cluster made up 12.9\% of cells analyzed and was enriched for genes belonging to the Shh signaling pathway (Fig. $2 \mathrm{G}$ ). Therefore, we named this cluster of cells Shh-responding progenitor cells, or ShhPCs.

We hypothesized that the SthhPC cluster (Fig. 3A) was similar to the $G s x_{2}{ }^{+}$tri-potent progenitor cluster identified by Zhang et al. (Zhang et al., 2020). Indeed, we found that Gsx 2 mRNA was enriched in ShhPCs (Fig. 3 B). We additionally identified several other differentially expressed genes enriched in the ShhPC cluster (Fig. 3B-C; Table S2), and others that were not 

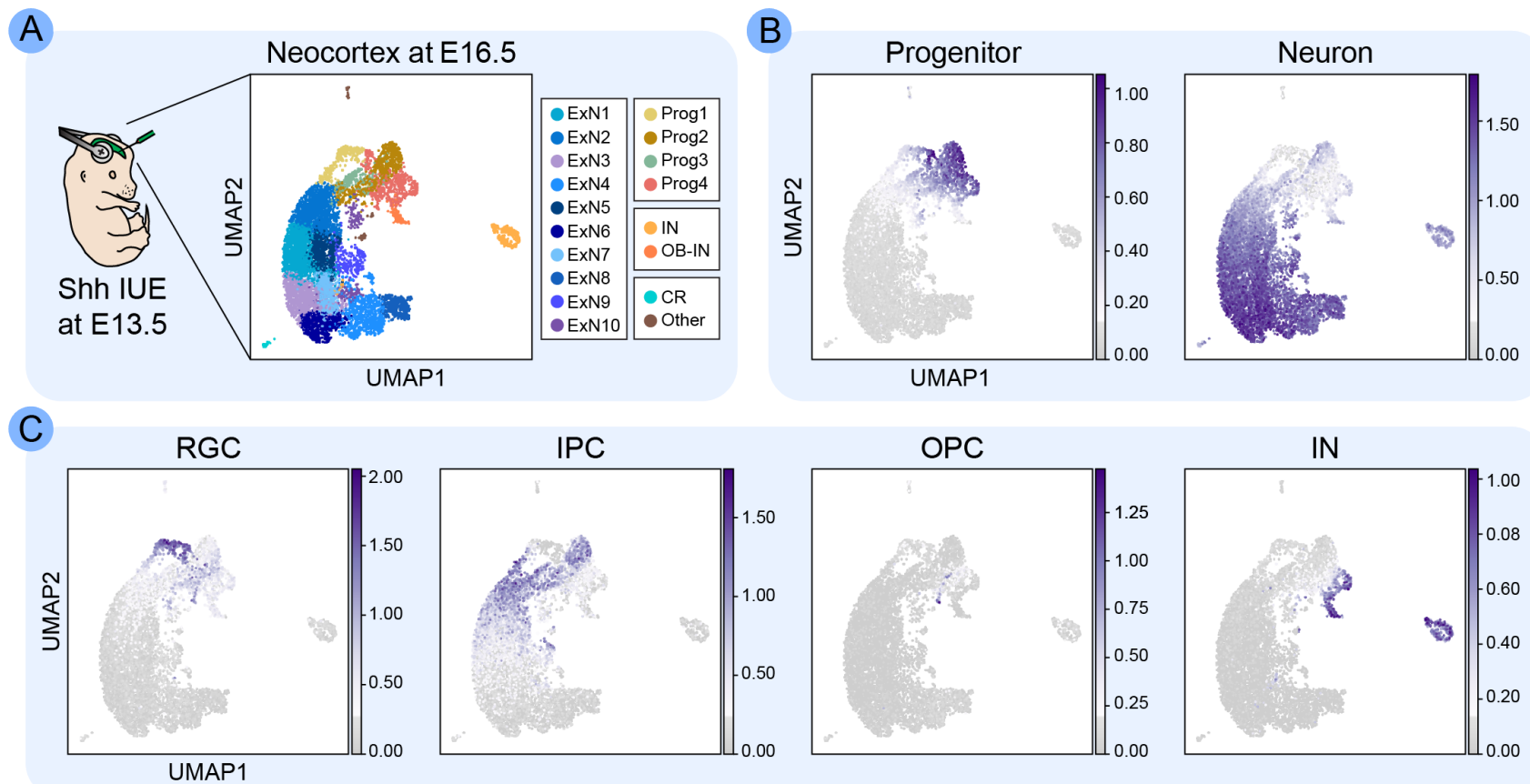

Fig. 1. Identification of Progenitor Cells. (A) We analyzed data generated by Zhang et al., in which they ectopically expressed Shh in the cortical VZ by electroporating progenitors with $p C A G-S h h N$-ires-GFP plasmid at E13.5 (Shh IUE) and performed scRNA-seq at E16.5. UMAP visualization of resulting cell clusters identified by Scanpy and annotated by major cell type. "Other" denotes microglia and endothelial cells. (B) UMAP of cells colored by mean expression of Progenitor and Neuron marker gene sets. (C) UMAP of cells colored by mean expression of RGC, IPC, OPC and IN marker gene sets. See also Table S1 for marker genes.

only enriched but also fairly restricted to ShhPCs (Fig. ${ }_{3} \mathrm{D}$; Table S2). Of particular interest among the ShhPC differentially expressed genes was the transcription factor Ascl1, which is in the same transcriptional network as Gsx2 (Waclaw et al., 2009; Wang et al., 2009). Ascl1 plays an important role in oligodendrogenesis in the spinal cord and ventral forebrain (Nakatani et al., 2013; Parras et al., 2007; Sugimori et al., 2007) and is one of the first marker genes in the OPC lineage (Nakatani et al., 2013; Vue et al., 2014).

Progenitor cluster relationships and lineage trajectories

To gain a deeper understanding of the relationships between progenitor clusters, we performed a heatmap and dendrogram analysis (Fig. 4A). We excluded cluster IPC1 as it was dominated by high expression of ribosome biogenesis genes, which confounded analysis. Based on hierarchical clustering predicted by the dendrogram, RGCs, ShhPCs, and OPCs were more closely related to each other than to OB-INs and IPCs (Fig. 4A). This is in line with ShhPCs and OPCs being more progenitor-like, whereas OB-INs and
IPCs are more neuronal. Within the progenitor-like branch, ShhPCs and OPCs were predicted to be more closely related to each other than to RGCs, which is supported by shared expression of genes like Ascl1.

To understand how the OPC lineage emerges during development, we focused on branches that included RGCs, ShhPCs, OPCs and OB-INs (Fig. 4B). We used RNA velocity (Bergen et al., 2020; La Manno et al., 2018) to predict lineage trajectories of cells in the different clusters (Fig. 4C). Projection of the velocities onto the progenitors indicated lineage progression from RGCs to IPCs, as expected (Fig. 4C). Additionally, RNA velocity analysis predicted cell-tocell transitions from ShhPCs to both OPCs and OBINs (Fig. $4 \mathrm{C}$ ). Plotting the velocity vector fields at single-cell resolution allowed us to predict future states of individual cells (Fig. 4D). Cells in the more differentiated clusters of IPCs, OPCs and OB-INs displayed strong directional RNA velocity flow toward the ends of their branches. Conversely, velocities of cells in the RGC and ShhPC clusters were smaller and less directional, reflecting their less committed state (Fig. 4D). Interestingly, some 


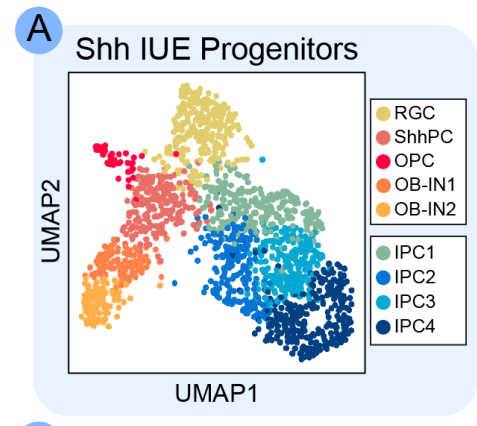

B
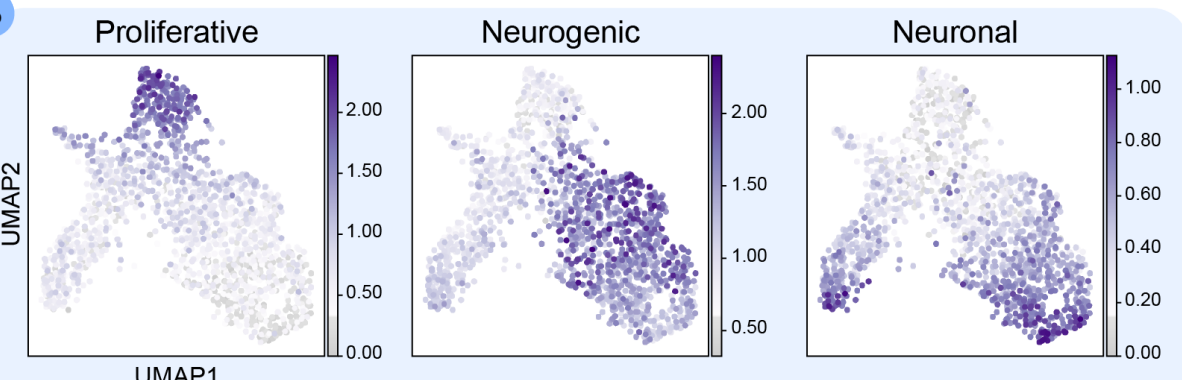

C
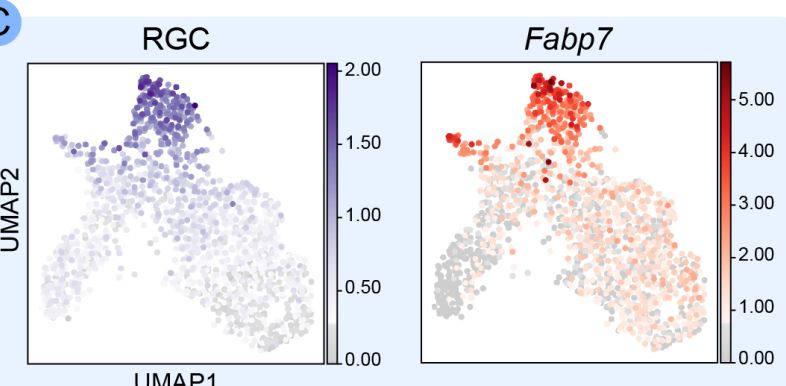

D
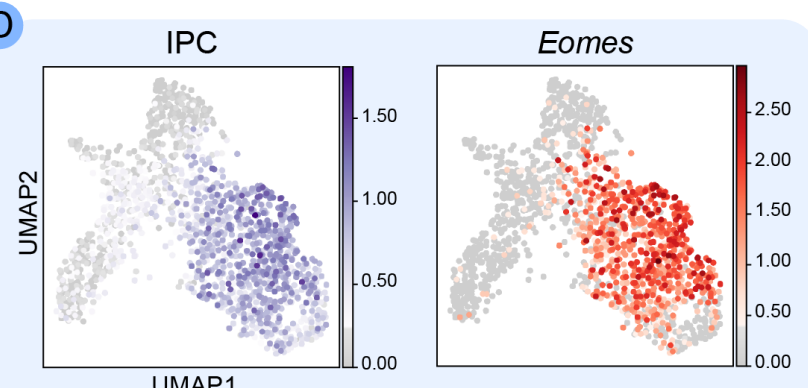

E
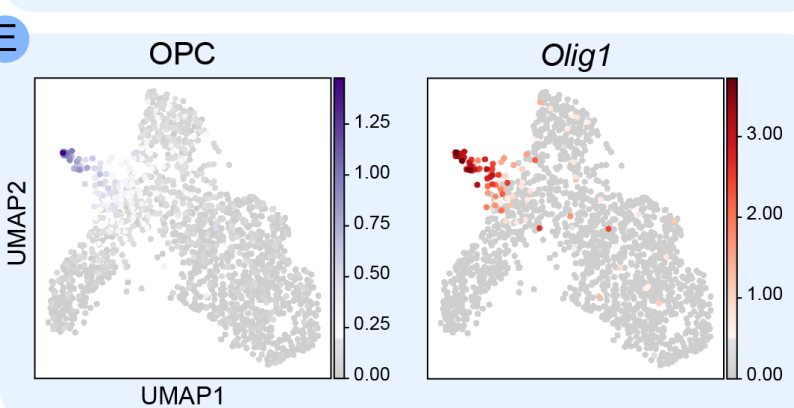

F
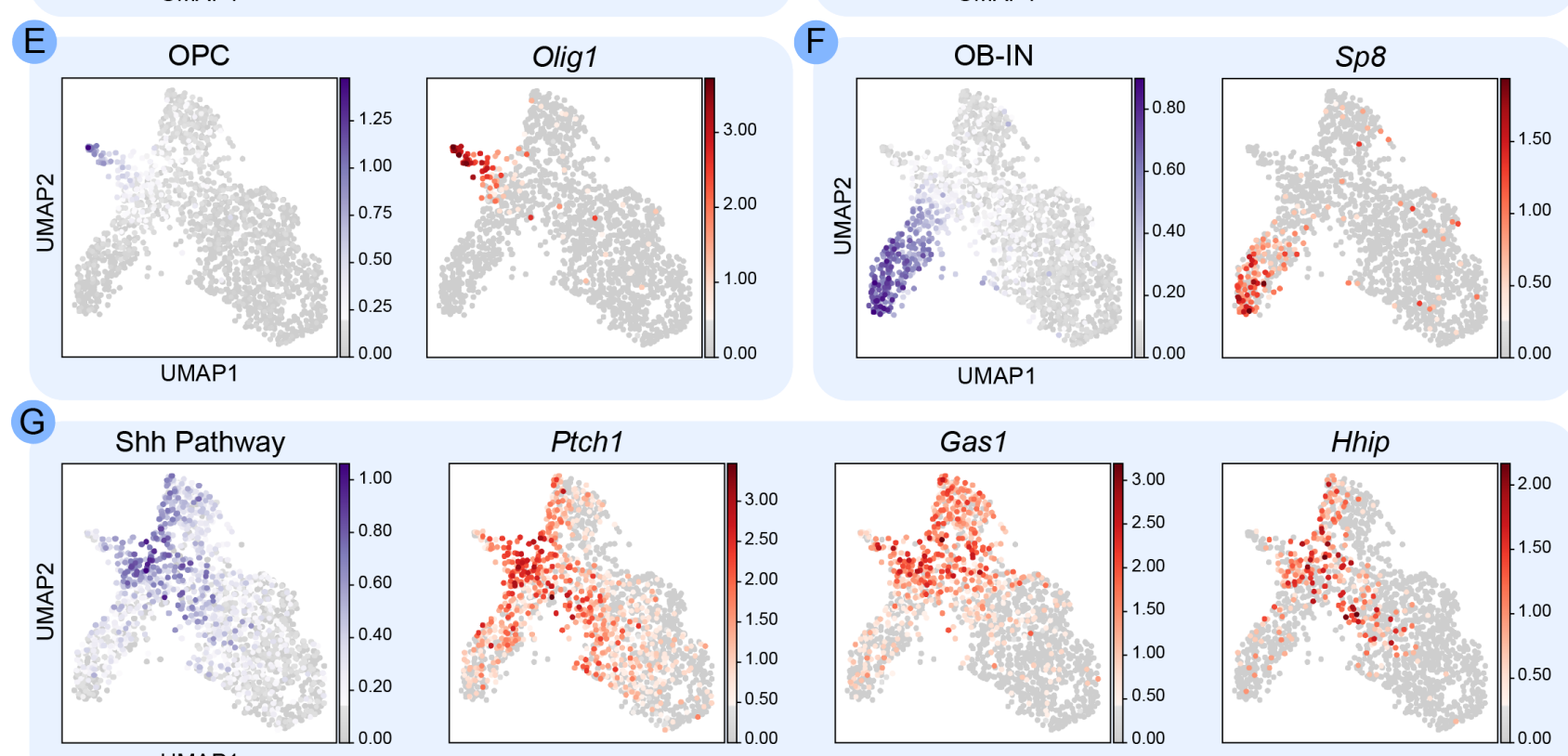

Fig. 2. Clustering the Progenitor Cells. (A) Scanpy clustering was performed on all progenitor cells and presumptive OB-INs. Nine clusters were identified and annotated based on gene expression: RGC, radial glial cells; IPC1-4, intermediate progenitor cells; OB-IN1-2, olfactory bulb interneurons; OPC, oligodendrocyte precursor cells; ShhPC, Shh-responding progenitor cells. (B) UMAP of cells colored by mean expression of Proliferative, Neurogenic and Neuronal markers. (C-F) UMAPs of cells colored by mean expression of either groups of cell type markers (blue) or individual canonical marker genes (red) for each cell type. (G) UMAP of cells colored by mean expression of Shh Pathway markers (blue) and mean expression of individual genes (red). See also Table S1 for marker genes.

ShhPCs had RNA velocities oriented toward OB-INs whereas others were directed toward OPCs (Fig. 4D), suggesting that the two lineages diverge at the ShhPC stage. We therefore performed lineage trajectory analysis using Slingshot (Street et al., 2018) on the RGC, ShhPC, OPC, and OB-IN clusters (Fig. 4B).
Slingshot predicted two distinct lineages emerging from the ShhPC cluster (Fig. 4E). Both lineages started at RGCs and transitioned through the ShhPC state, at which point they diverged to either OPCs or OB-INs (Fig. 4E). Aligning predicted Slingshot lineages along pseudotime indicated that ShhPCs 

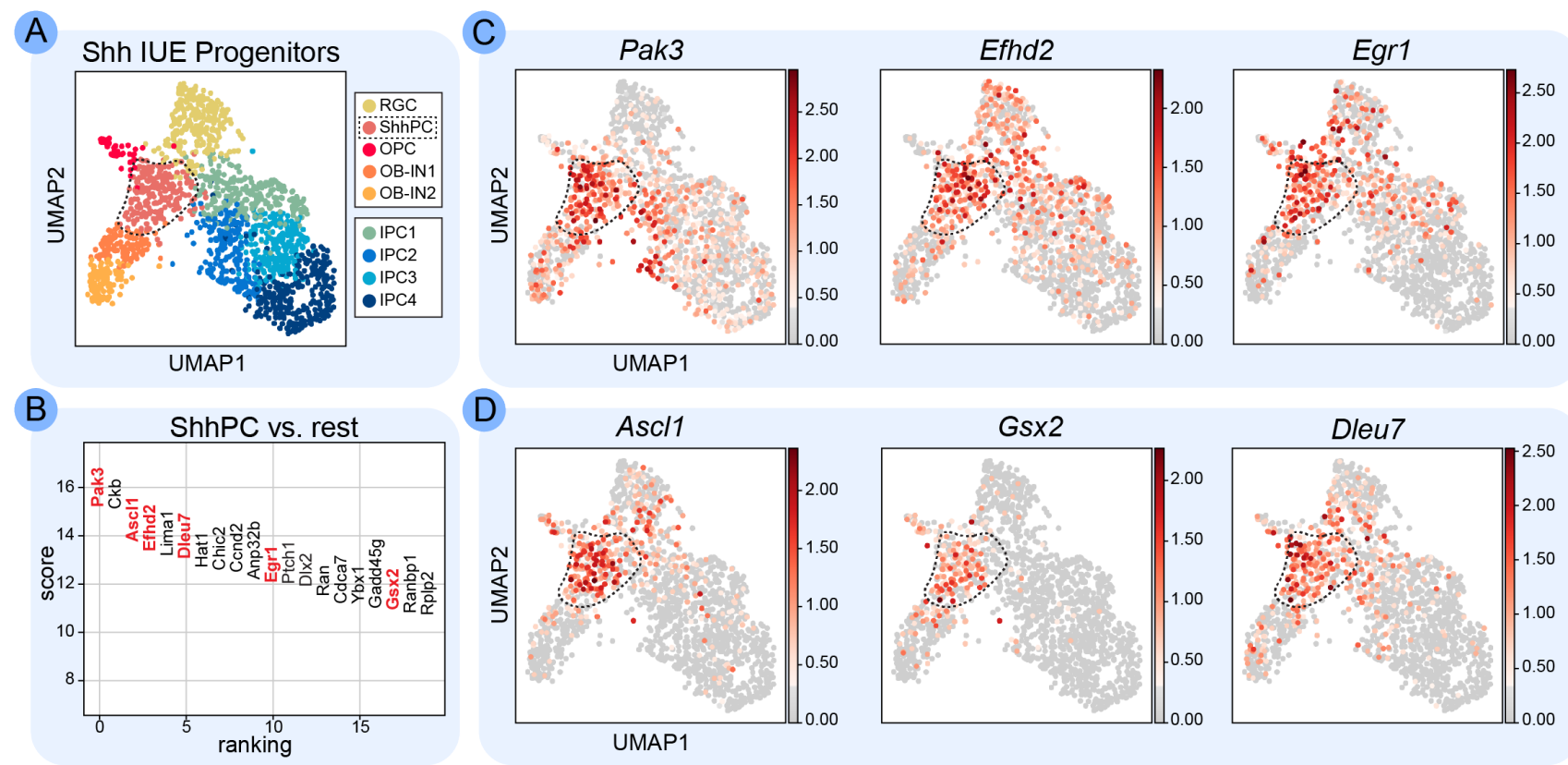

Fig. 3. Differentially Expressed Genes in the Shh Progenitor Cluster. (A) UMAP of all progenitor cells and OB-INs, highlighting the ShhPC cluster. (B) Genes that are differentially expressed in the ShhPC cluster compared to all other clusters. Genes with corresponding UMAPs in (C) and (D) are shown in red. (C) UMAP of cells colored by mean expression of Pak3, Efhd2 and Egr1, which are all enriched in the ShhPC cluster. (D) UMAP of cells colored by mean expression of Ascl1, Gsx2 and Dleu7, which are all more restricted to the ShhPC cluster. See also Table S2 for ShhPC-enriched genes.

were between RGCs and the more differentiated OBIN or OPC clusters (Fig. $4 \mathrm{E}$ ), reinforcing the idea that ShhPCs are progenitors that are committing to these two lineages in response to Shh signaling.

We next employed tradeSeq (Van den Berge et al., 2020) to identify differentially expressed genes between the OPC and OB-IN lineages over pseudotime (Fig. 5, Table $S_{3}$ ). We focused our analysis between tradeSeq knots 2 and 5 (see Methods), as this incorporated ShhPCs and the predicted divergence of the OPC and OB-IN lineages (Fig. 5A). Estimated smoother curves for the known OPC-specific gene, Olig1, showed upregulated and stabilized expression in the OPC lineage over pseudotime (Fig. 5 B). Conversely, smoother curves for the OB-IN specification gene, $S p 8$, indicated sustained expression in the OB-IN lineage (Fig. ${ }_{5} \mathrm{~B}$ ). Beyond these well-characterized examples of OPC- or OB-IN-specific markers, we identified several genes expressed in ShhPCs that either stayed on or were further upregulated in the OPC lineage, but not in the OB-IN lineage (Fig. 5C). Interestingly, Ascl1 and Gsx2 were maintained in newly formed OPCs but downregulated in newly formed OB-INs (Fig. $5 \mathrm{C}$ ), implicating these transcription factors specifically in the OPC lineage. In contrast, several other genes were upregulated or maintained in the OB-IN lineage and downregulated in the OPC lineage (Fig. 5D). These included genes important for OB-IN fate determination, such as the transcription factors $S p 9$, Pax6 and Dlx1 (Fig. 5D). Together, these lineage analyses allowed us to identify the transcriptional changes conferred by Shh signaling that prime a subset of dorsal forebrain progenitors to acquire OPC or OB-IN fates.

Given the clear split in gene expression over pseudotime between the OPC and OB-IN lineages, we further subclustered the ShhPCs to identify any subpopulations (Fig. 6A). We found 3 distinct ShhPC subclusters: ShhPC1, ShhPC2, and ShhPC 3 . Similar to cluster IPC1, ShhPC 3 was dominated by high expression of genes involved in ribosome biogenesis. Therefore, we focused on ShhPC1 and ShhPC2. Enrichment analysis of ShhPC1 found overrepresentation of genes with gene ontology (GO) terms associated with gliogenesis, glial fate commitment, and oligodendrocyte differentiation (Fig. 6B, Tables $\mathrm{S}_{4}$ and $\mathrm{S}_{5}$ ). Alternatively, ShhPC2 had 


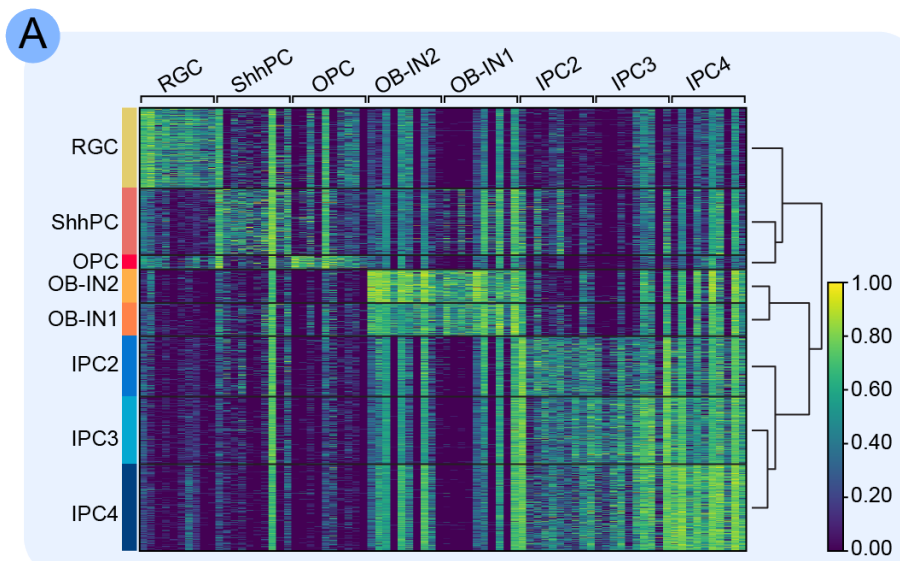

B

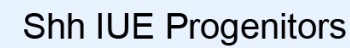

C

RNA Velocity Vector Field

D
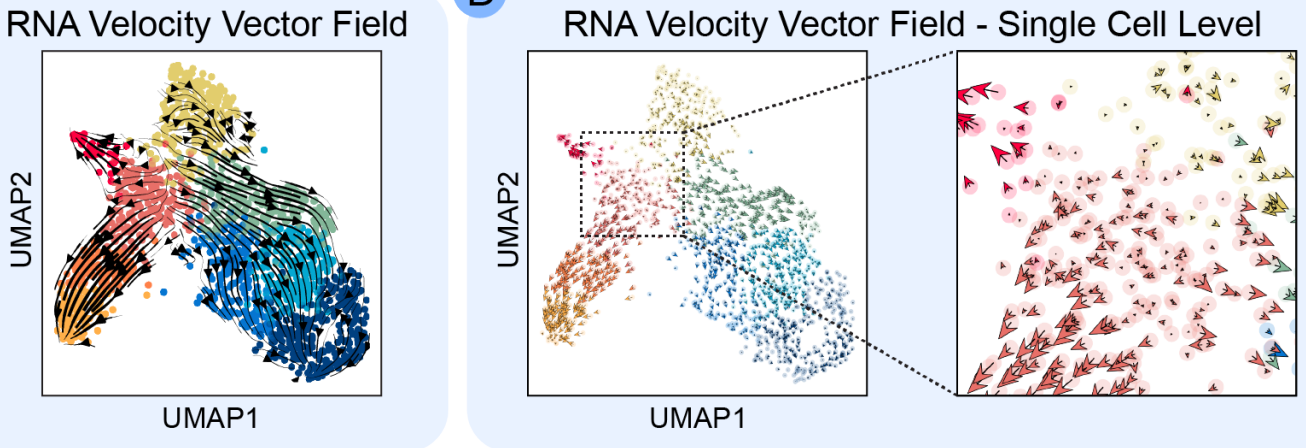

E
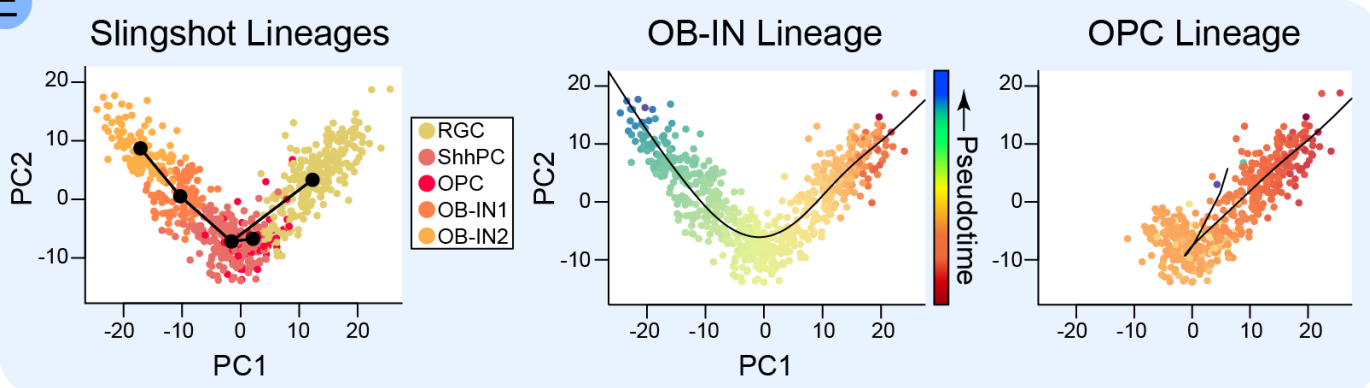

Fig. 4. Lineage Analysis of OPCs and OB-INs. (A) Heatmap showing marker gene expression for the top genes of each cluster. Each column represents expression of one gene, and each row represents expression in one cell. Dendrogram shows hierarchical clustering. (B) UMAP of all progenitor cells and OB-INs, highlighting the arm that includes the RGC, ShhPC, OB-IN1, OB-IN2, and OPC clusters. (C) Velocities derived using the scVelo stochastic model, projected onto the UMAP. The main gene-averaged flow visualized by velocity streamlines shows a split between the IPC clusters and the OPC, OB-IN1 and OB-IN2 clusters, which are all derived from progenitors in the RGC and ShhPC clusters. (D) Fine-grained resolution of the velocity vector field in (C) shown at single-cell level Each arrow represents the direction and speed of movement of an individual cell. (E) Lineages of the clusters highlighted in (B), as predicted by Slingshot. Slingshot predicts that OPC and OB-IN lineages bifurcate at the ShhPC cluster. Cells are colored by cluster (left) or by pseudotime (middle and right). Note that the looping in the OPC lineage indicates that the endpoint would be coming into or out of the XY plane if presented in 3 dimensions.

over-representation of genes with GO terms associated with neuron fate commitment and interneuron differentiation (Fig. 6B, Tables S6 and $\left.\mathrm{S}_{7}\right)$. Further examination of specific gene expression reflects this heterogeneity (Fig. 6C); ShhPC1 had higher expression in genes associated with Notch signaling (Hes1, Notch1), Shh signaling (Ptch1, Hhip,
Gas1), and OPC function (Olig2, Pdgfra) compared to ShhPC2, which showed higher expression in genes associated with the OB-IN lineage (Dlx1, Dlx2, Arx, Sp9). Interestingly, ShhPC1 shared some weak gene expression with RGCs (Ptprz1, Hes1, Notch1), while ShhPC2 shared Gadd45g expression with IPCs, perhaps indicating that ShhPC1 cells retain more 

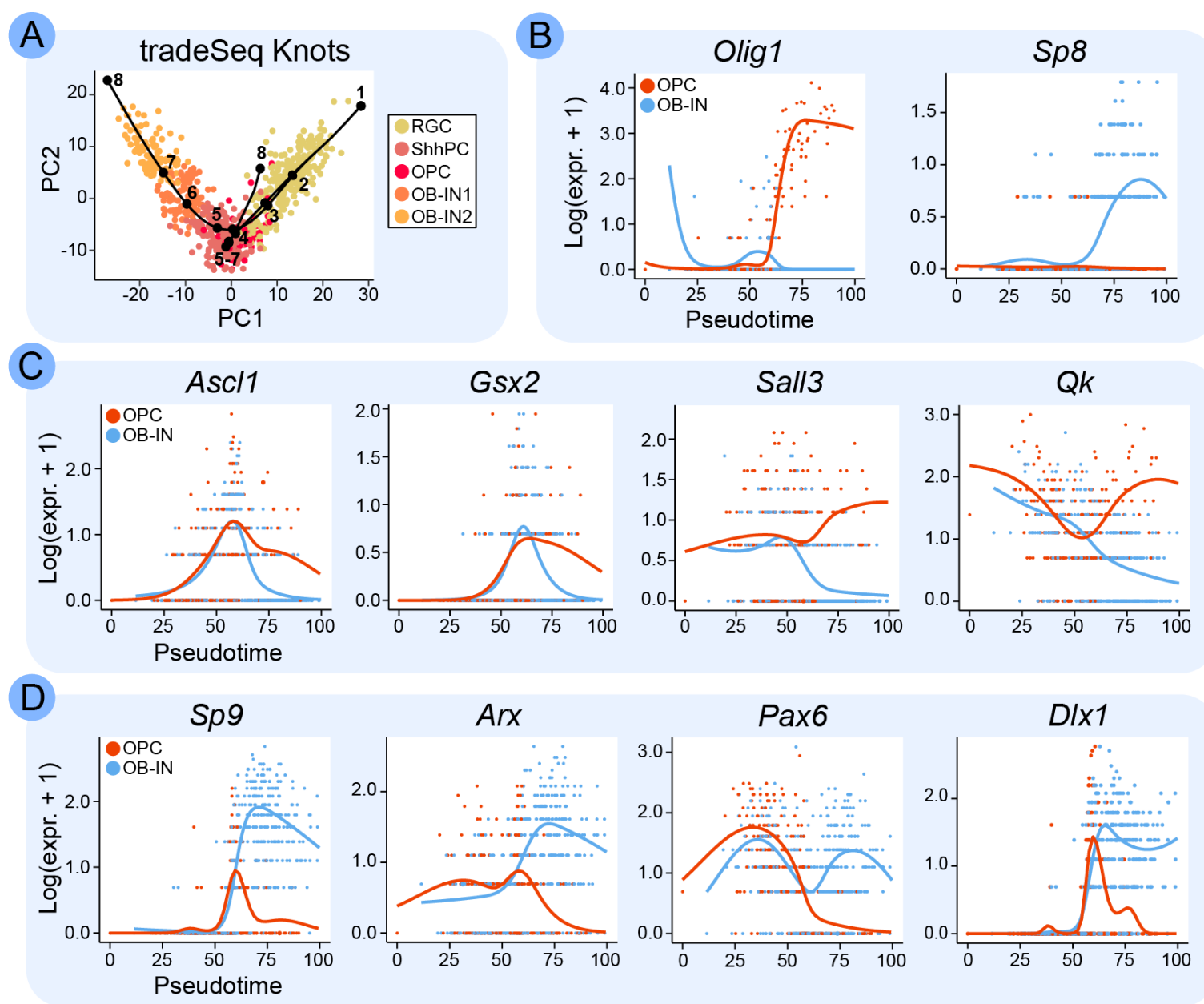

Fig. 5. Differentially Expressed Genes Between OPC vs. OB-IN Lineages. (A) PCA plot for lineage trajectories predicted by Slingshot, illustrating the 8 knots used for tradeSeq analysis. tradeSeq was used to identify genes differentially expressed between the OPC and OB-IN lineages between knots 2 and 5 . Cells are colored by cluster. (B) Estimated smoothers for Olig1 and Sp8, as predicted by tradeSeq. (C) Estimated smoothers for example OPC lineage-specific genes predicted by tradeSeq. Ascl1, Gsx2, Sall3, and Qk all have predicted expression in ShhPCs, and continued expression in the OPC lineage over pseudotime. (D) Estimated smoothers for example OB-IN lineage-specific genes predicted by tradeSeq. Sp9, Arx, Pax6, and DIx1 all have predicted expression in ShhPCs, and continued expression in the OB-IN lineage. See also Table S3 for tradeSeq results.

RGC-like characteristics and ShhPC2 cells are more IPC-like (Fig. 6C).

Together, our analyses indicate that increased Shh signaling can instruct dorsal forebrain RGCs towards a Shh-responsive progenitor cell fate, and that these ShhPCs can generate OPC and OB-IN lineages. We identified pre-OPCs as a subpopulation of ShhPCs that appear to be committed to the oligodendrocyte lineage and precede OPCs based on gene expression: pre-OPCs express genes shared between all ShhPCs in addition to some that are more restricted. Thus, we were able to generate a combinatorial gene expression signature for pre-OPCs.
Identification of pre-OPCs in wild-type brains

Because these data involved ectopic expression of Shh, we wondered whether the pre-OPC signature could be found in normally developing brains. Therefore, we analyzed scRNA-seq data collected from the neocortices of wild-type mice at different embryonic ages (Fig. 7). We first analyzed a dataset from E18.0 dorsal forebrains (La Manno et al., 2020), since this is a stage when dorsally-derived cells in the OPC lineage are abundant (Winkler et al., 2018). We clustered all cells based on gene expression to identify progenitors (Fig. 7A), then focused our analysis on just the progenitor clusters. Classification of progenitor clusters based on gene expression profiles identified 


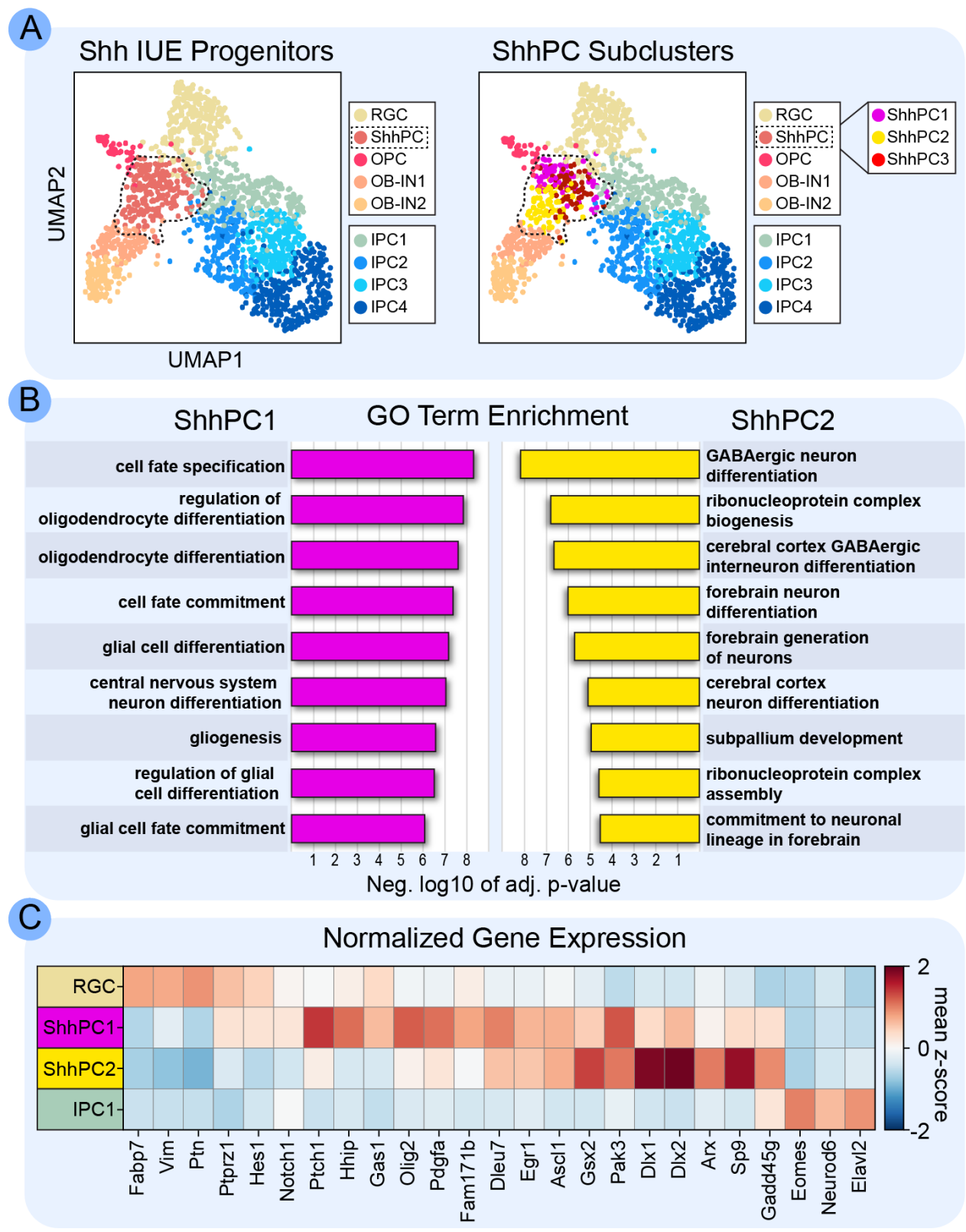

Fig. 6. Subclustering the Shh Progenitor Cluster. (A) UMAP of all progenitor cells and OB-INs, highlighting the ShhPC cluster (left). The ShhPC cluster subclustered into three distinct subclusters (right): ShhPC1, ShhPC2, and ShhPC3. (B) Functional annotation of differentially expressed genes identified by Scanpy show top Gene Ontology (GO) term biological processes associated with ShhPC1 and ShhPC2. (C) Heatmap showing expression of specific genes of interest between ShhPC1 and ShhPC2, using RGC and IPC1 as control comparisons. See also Tables S4-7 for enriched genes and GO term results.

RGCs, IPCs, INs, astrocytes and OPCs (Fig. 7A-B). Overlaying our putative pre-OPC signature clearly identified clusters with high expression of this gene set (Fig. $7 \mathrm{~B}$ ). Cells with a strong pre-OPC gene expression signature were adjacent to cells in the RGC and OPC clusters that exhibited weaker expression of pre-OPC genes (Fig. $7 \mathrm{~B}$ ). Individual genes from the pre-OPC signature were also enriched, including Ascl1, Gsx2, Egfr and $\mathrm{Sall}_{3}$ (Fig. 7C). These data indicate that the
pre-OPC transcriptional profile is present in a subset of progenitors in the E18.o wild-type dorsal forebrain.

We next analyzed scRNA-seq data from E16.5 (Fig. 7D-E) (La Manno et al., 2020) and E15.5 (Fig. 7 F-G) (Yuzwa et al., 2017). Very few OPCs are present at these timepoints, but a cluster of progenitor cells at each age exhibited robust expression of genes in the pre-OPC signature (Fig. $7 \mathrm{E}, \mathrm{G}$ ). At both ages, 
bioRxiv preprint doi: https://doi.org/10.1101/2020.10.18.344515; this version posted October 18, 2020. The copyright holder for this preprint (which was not certified by peer review) is the author/funder, who has granted bioRxiv a license to display the preprint in perpetuity. It is made available under aCC-BY-NC-ND 4.0 International license.

A

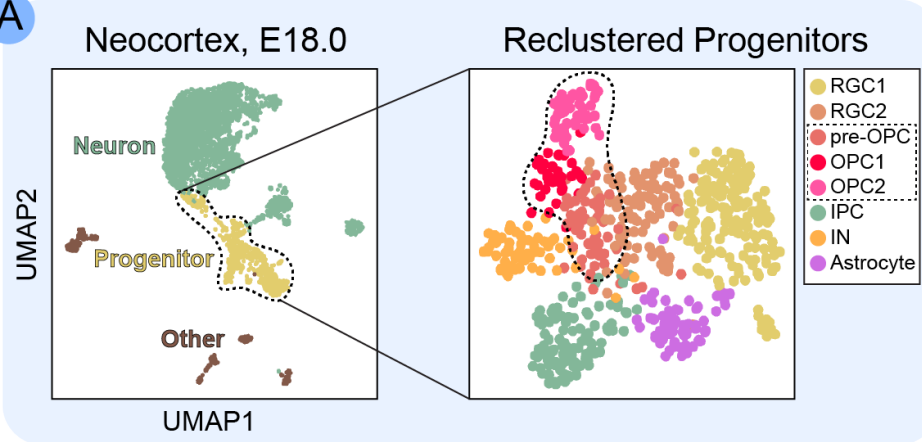

B

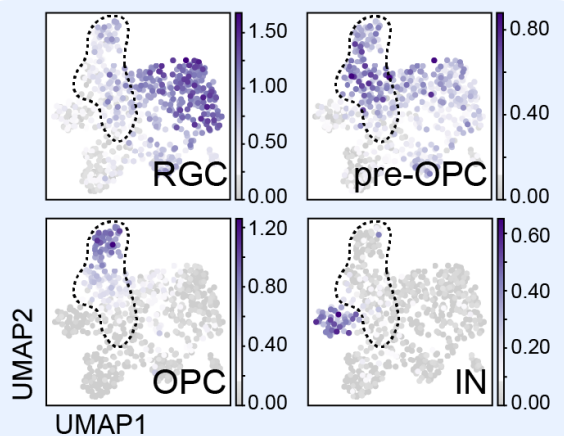

C
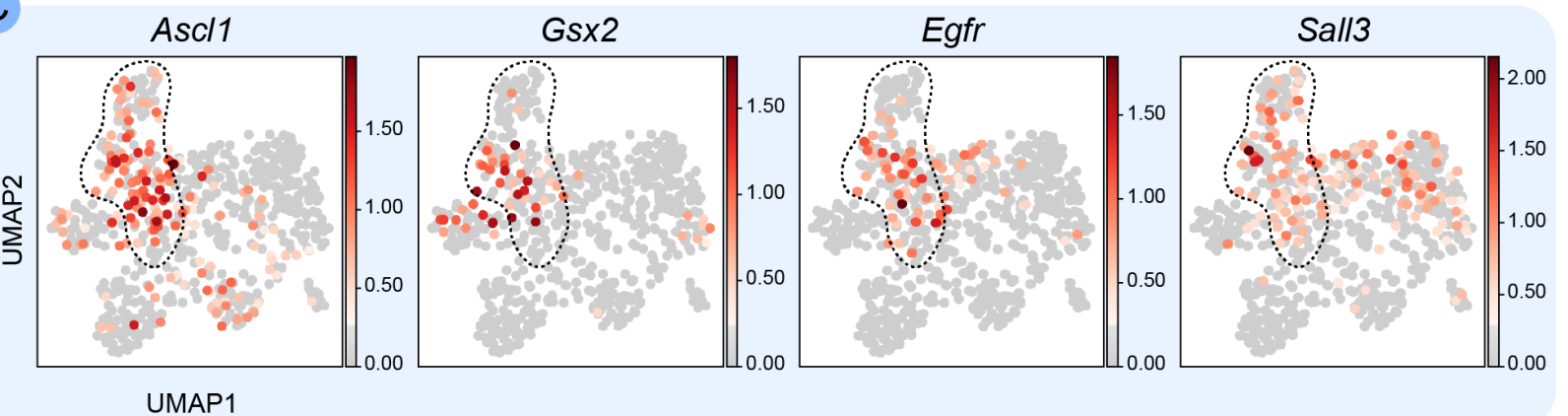

(D)

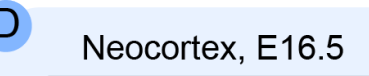

Reclustered Progenitors
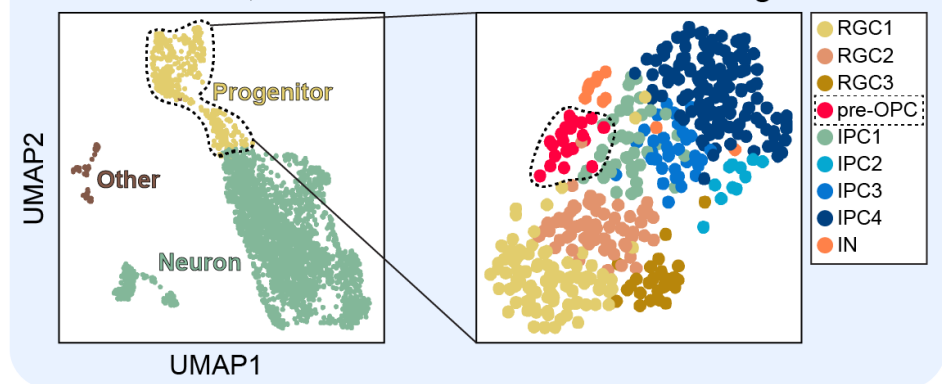

\section{F}
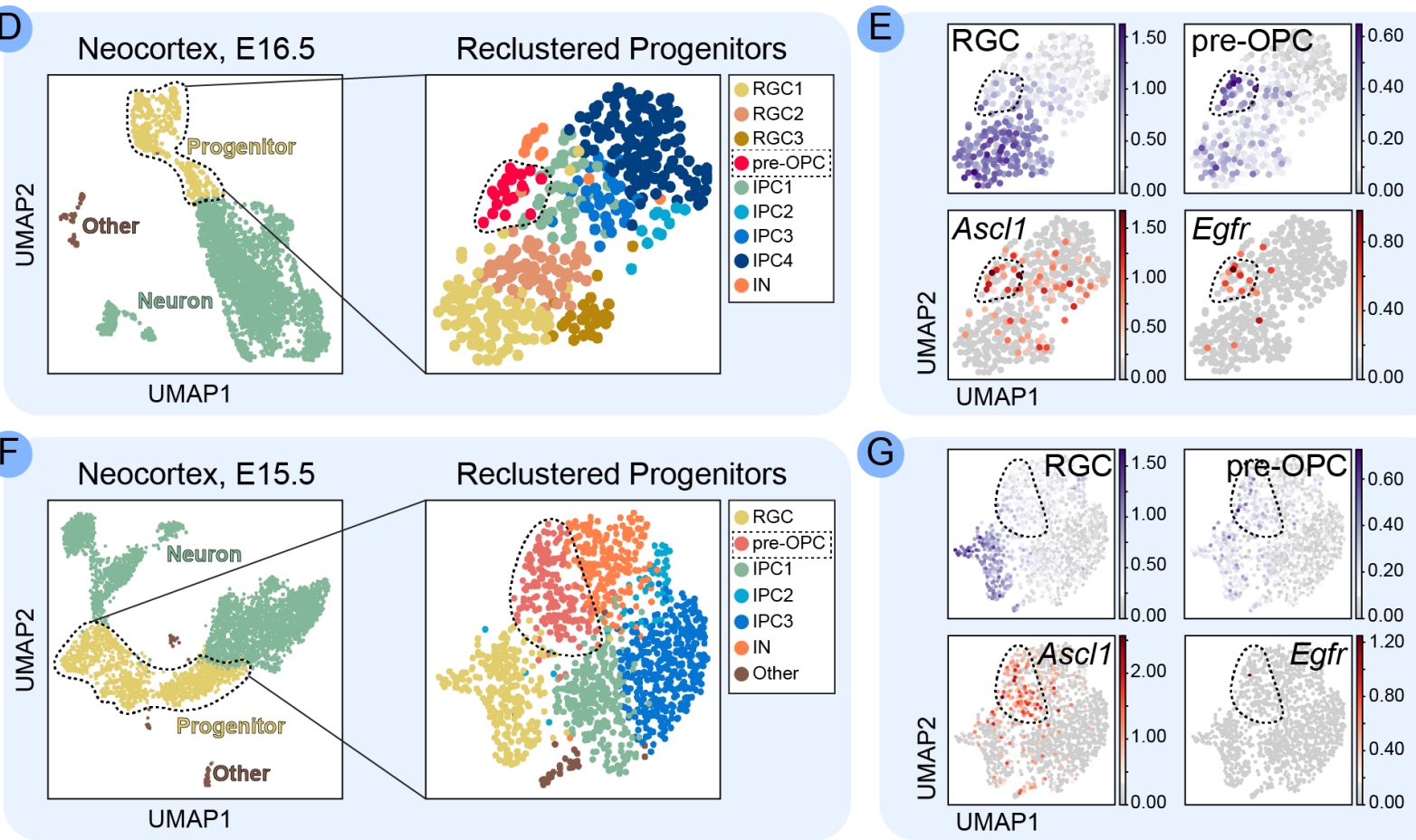

G

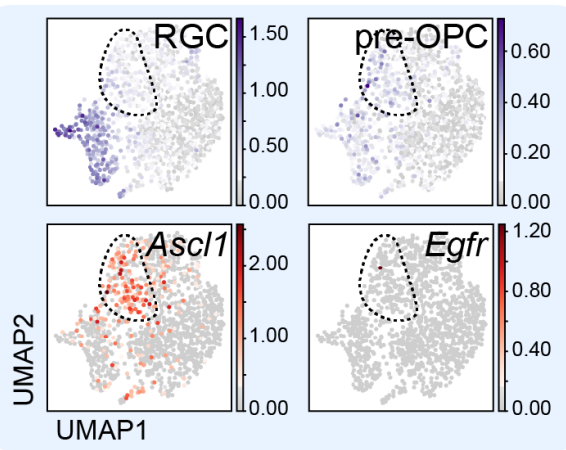

Fig. 7. Pre-OPC Progenitors in Normal Development. (A) Scatterplot of cells after principal-component analysis and UMAP visualization of E18.0 wildtype (WT) neocortex. Left panel: all cells collected for ScRNA-seq analysis. Right panel: new principal-component analysis and UMAP visualization of just the progenitor clusters. Eight progenitor clusters were identified and annotated based on gene expression: radial glial cells (RGC1-2), oligodendrocyte precursor cells (pre-OPC, OPC1-2), intermediate progenitor cells (IPC), interneurons (IN) and Astrocytes. Cells are colored according to Scanpy clustering. (B) UMAP of E18.0 progenitor cells colored by mean expression of RGC, pre-OPC, OPC and IN marker gene sets. (C) UMAP of E18.0 progenitor cells colored by mean expression of specific pre-OPC genes identified in the E16.5 Shh-IUE dataset. (D) Same as in (A), except with E16.5 WT neocortex data. (E) UMAP of E16.5 progenitor cells colored by mean expression of RGC and preOPC marker gene sets (blue), and by mean expression of Ascl1 and Egfr (red). (F) Same as in (A) except with E15.5 WT neocortex data. (G) Same as in (E) except with E15.5 progenitor cells. For all UMAPs, "Other" denotes microglia and endothelial cells. 
expression of Ascl1 was enriched in the putative preOPC clusters (Fig. 7 E, G). Egfr was previously identified as being enriched early in OPC development (Falcão et al., 2018; Huang et al., 2020; La Manno et al., 2020; Marisca et al., 2020; Marques et al., 2016). We found that Egfr expression was enriched in pre-OPCs at E18.5 (Fig. $7 \mathrm{C}$ ) and E16.5 (Fig. $7 \mathrm{E}$ ) but was not yet strongly expressed at E15.5 (Fig. $7 \mathrm{G}$ ). Thus, Ascl ${ }^{+}$;Egfr progenitors at E15.5 could be ShhPCs that have not yet committed to the pre-OPC lineage, which would be consistent with studies from our lab that dorsal progenitors start to give rise to OPCs between E15.5-E17.5 (Winkler et al., 2018).

Validation of Ascl1 as a pre-OPC marker in the embryonic dorsal forebrain

Previous studies demonstrated important roles for Ascl 1 in OPC development in the spinal cord (Battiste et al., 2007; Kelenis et al., 2018; Sugimori et al., 2007; Sugimori et al., 2008; Vue et al., 2014), embryonic ventral forebrain (Parras et al., 2007; Yung et al., 2002) and postnatal telencephalon (Nakatani et al., 2013; Parras et al., 2004). These studies indicate that Ascl1 is already expressed early in OPC development (Battiste et al., 2007; Parras et al., 2007; Sugimori et al., 2007). To validate our scRNA-seq analysis, we performed multiplex mRNA in situ hybridization to detect Ascl1 mRNA and the dorsal progenitor marker, Emx1 (Fig. 8A-B). At E15.5 Emx1 was highly expressed in the

dorsal progenitor zones, while Ascl1 expression was strongest in ventral progenitors (Fig. 8A). However, we could readily detect low levels of Ascl1 in the $E m x 1^{+} \mathrm{VZ}$, where dorsal progenitors reside (Fig. 8A). By E16.5, Ascl1 expression was even more evident in the $E m x 1^{+}$dorsal VZ, although it was still weaker than in ventral regions (Fig. $8 \mathrm{~B}$ ). We next performed immunohistochemistry for Ascl1 protein in E16.5 Emx1-Cre;NZG mice (Winkler et al., 2018), in which dorsal RGCs and their progeny express nuclear $\beta$-gal (Fig. 8C). We detected Ascl1; $\beta$-gal double-positive cells in the ventricular and subventricular zones, further confirming Ascl1 expression in dorsal progenitors. Ascl 1 expression was weak in the $\mathrm{VZ}$ and stronger in more basal cells (Fig. 8C), suggesting expression is initiated in RGCs. Interestingly, apical cells that were weakly positive for Ascl1 were usually negative for Olig2, whereas the higher-expressing basal Ascl1 ${ }^{+}$cells often co-expressed Olig2 (Fig. 8C). This pattern is consistent with Ascl1 expression being initiated in RGCs before Olig2 expression starts, prior to acquisition of an OPC fate. We also identified a subset of basally-located cells strongly expressing Ascl1 but not Olig2 (Fig. 8C), which could represent $\mathrm{Ascl}^{+}{ }^{+}$cells fated to make OB-INs instead of OPCs.

Lineage-tracing studies using Ascl1-Cre and Ascl1CreERT2 mice demonstrated that $\mathrm{Ascl}^{+}$progenitors give rise to many different cell types in the brain, including oligodendrocytes and OB-INs, but not neocortical excitatory neurons (Kelenis et al., 2018; Kim et al., 2008; Kim et al., 2011). However, these studies did not determine which cell types derived from $\mathrm{Ascl}^{+}$progenitors specifically in the dorsal forebrain. To test if dorsal forebrain $\mathrm{Ascl}_{1}{ }^{+}$ progenitors can generate neocortical oligodendrocytes, we lineage traced these progenitors using in utero electroporation of Ascl1-CreERT2 embryos (Fig. 9). We crossed Ascl1-CreERT2 mice (Kim et al., 2011) to the cre-reporter strain, Ai9 (Madisen et al., 2010). We then used directional in utero electroporation of a piggyBac transposase-based plasmid (Garcia-Moreno et al., 2014) to achieve stable integration of a fluorescence reporter transgene (nuc.mT-Sapphire) specifically into dorsal RGCs at E15.5 (Fig. 9A). Immediately after in utero electroporation, we injected the pregnant dam with tamoxifen to label the Ascli $1^{+}$lineage with tdTomato (Fig. 9A). In this experiment, cells that are nuc.mTSapphire;tdTomato double-positive are specifically from the dorsal $\mathrm{Ascl}_{1}{ }^{+}$lineage. At postnatal day Po, the majority of electroporated nuc.mTSapphire;tdTomato double-positive cells were located in the SVZ (Fig. 9B), although cells could be found throughout the neocortex. We could readily detect many nuc.mT-Sapphire;tdTomato double-positive that also expressed Olig2 (Fig. 9B, arrows), demonstrating that dorsal $\mathrm{Ascl}^{+}$progenitors can generate oligodendrocytes. We also identified a smaller number of nuc.mT-Sapphire;tdTomato double-positive cells that did not express Olig2 (Fig. ${ }_{9} \mathrm{~B}$, arrowhead). The identity of these cells is not yet known, but they could be newborn OB-INs or possibly pre-OPCs that have not yet turned on Olig2 expression. Quantification of nuc.mTSapphire;tdTomato double-positive cells showed that $68.64 \%( \pm 3.48 \%, \mathrm{SEM})$ were Olig2 ${ }^{+}$, indicating that 


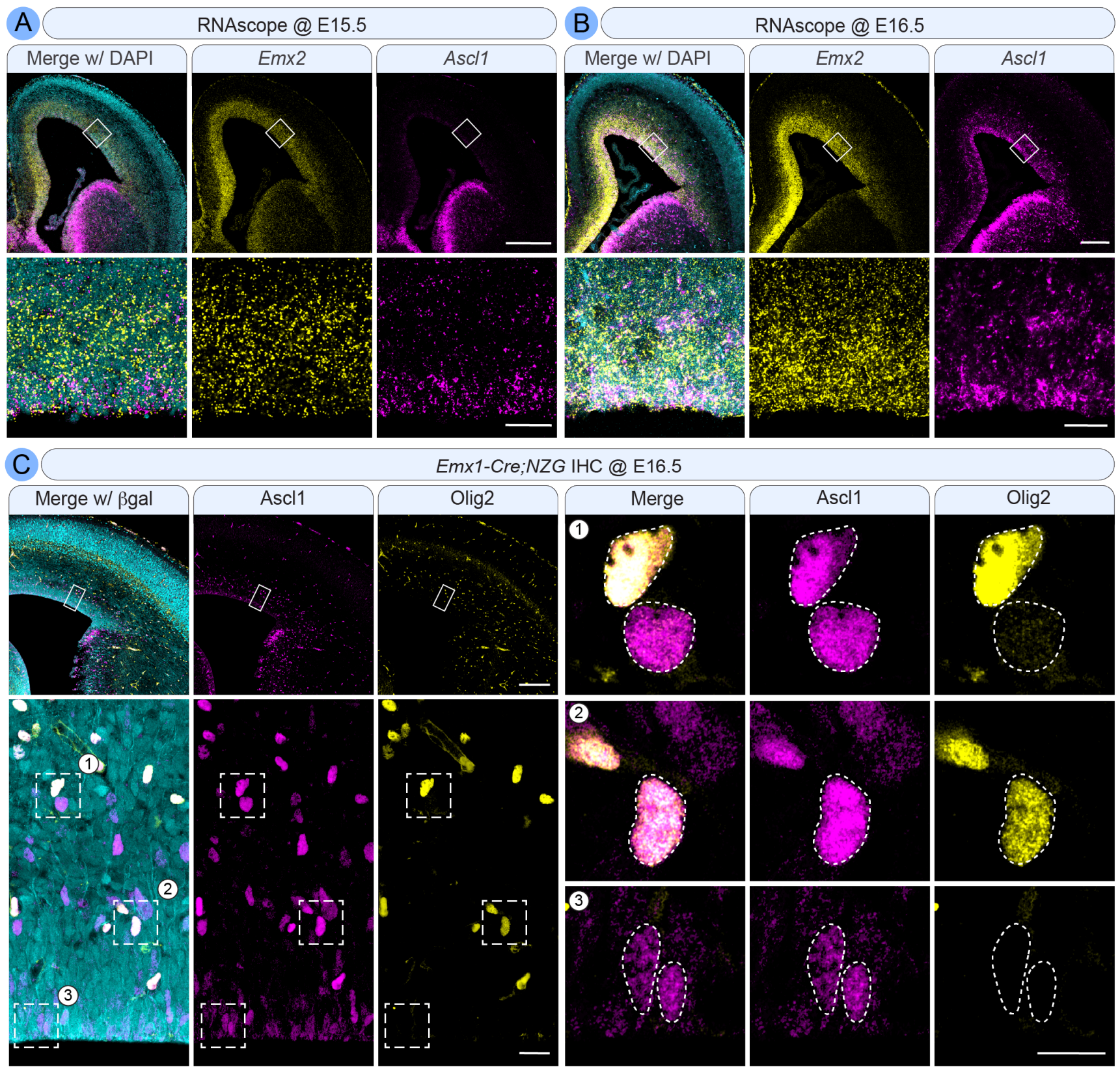

Fig. 8. Expression of Ascl1 mRNA and Ascl1 protein in $E m \times 1^{+}$dorsal progenitors. (A-B) RNAscope® assay to analyze mRNA levels of Emx2 (yellow) and Ascl1 (magenta) in the neocortex at E15.5 (A) and E16.5 (B), counterstained with DAPI (cyan) for nuclei. Top rows: overviews showing Emx1 expression is highest in dorsal progenitor domains, whereas Ascl1 is enriched in ventral regions. Bottom rows: higher magnification of the boxed areas showing Ascl1 is also expressed in the $E m \times 1^{+}$dorsal progenitor domain, and appears to increase from E15.5 (A) to E16.5 (B). Scale bars: $200 \mu \mathrm{m}$ overview (top rows), $20 \mu \mathrm{m}$ zoom (bottom rows). (C) Immunohistochemistry to analyze Ascl1 (magenta) and Olig2 (yellow) protein at E16.5 in Emx1-Cre;NZG reporter mice. $\beta$-gal (cyan) labels all cells from the Emx1+ dorsal progenitor lineage. Upper left panels: an overview of the imaged region. Bottom left panels: higher magnification of the boxed regions in the overviews, to identify $\beta$ gal/Ascl1 double-positive cells (numbered boxes). Panels 1-3 on the right are zoomed-in images of the corresponding numbered boxes on the bottom left, showing: 1) Two Ascl1 ${ }^{+}$nuclei in the upper subventricular zone, one co-expressing high levels of Olig2 and the other Olig2-negative; 2) Two Ascl1 ${ }^{+}$nuclei in the lower subventricular zone, both expressing moderate levels of Olig2; 3) Two nuclei in the ventricular zone weakly expressing Ascl1, both Olig2-negative. Scale bars: $200 \mu \mathrm{m}$ overview (top left row), $20 \mu \mathrm{m}$ zoom (bottom left row), $10 \mu \mathrm{m}$ individual nuclei (right). 

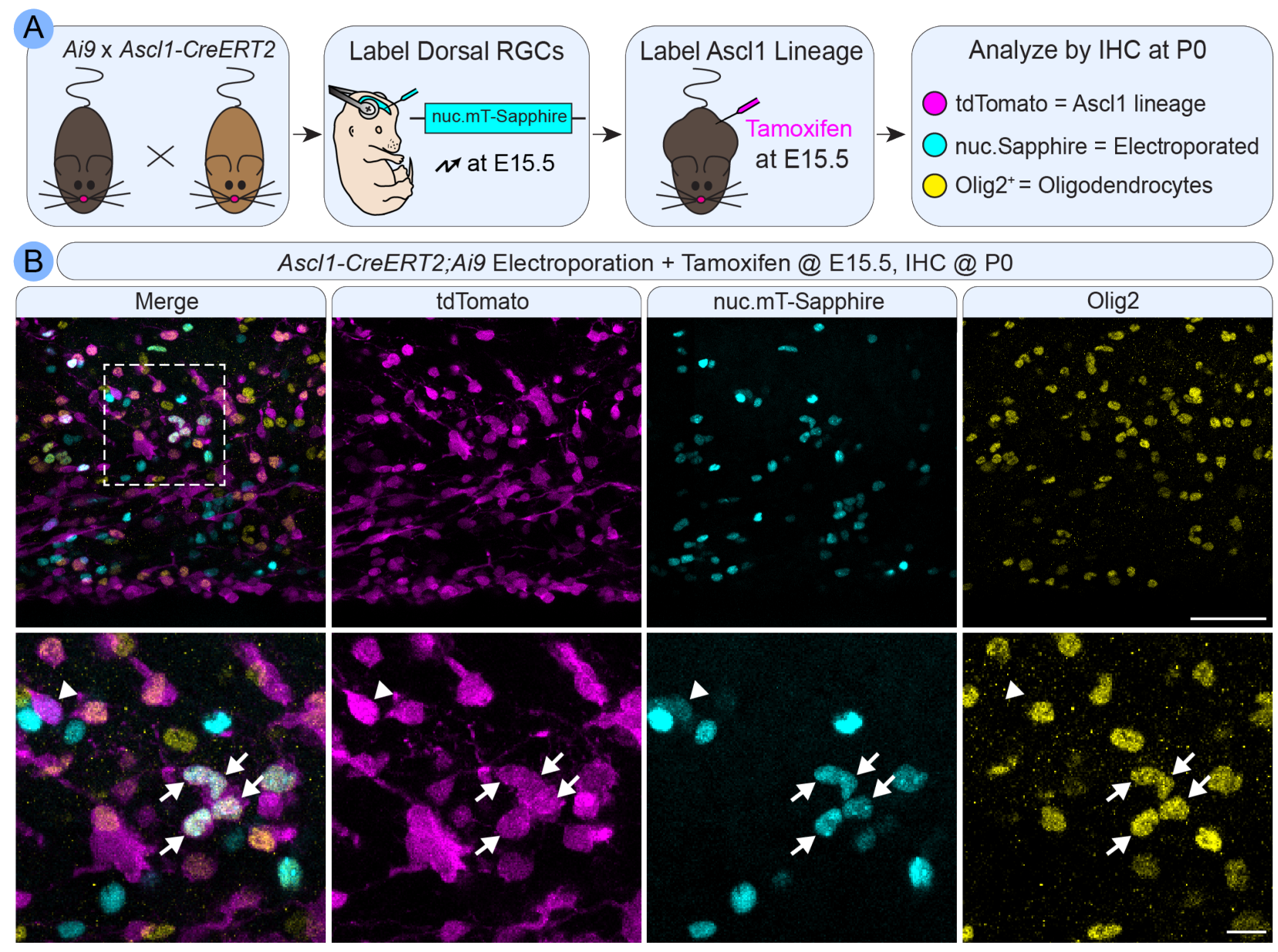

Fig. 9. Dorsal Ascl1+ cells can make OPCs. (A) Schematic of the genetic lineage-tracing approach to characterize dorsally-derived Ascl1-lineage cells in the neocortex. Ascl1 $1^{+/ c r e E R T 2}$ mice were crossed to $A i 9^{f / / f l}$ reporter mice. At E15.5, we used in utero electroporation to label dorsal RGCs and their offspring with nuc.mTSapphire. We then injected tamoxifen to permanently label all Ascl1 ${ }^{+}$progenitors and their offspring with tdTomato. Therefore, tdTomato/nuc.mT-Sapphire double-positive cells were from the dorsal Ascl1 lineage. Brains were collected at P0 and stained for Olig2 to label OPCs. (B) Immunohistochemical analysis of electroporated brains at P0. Top row, overview of the ventricular/subventricular zones showing tdTomato (magenta), nuc.mT-Sapphire (cyan) and Olig2 (yellow). Scale bar: $50 \mu \mathrm{m}$. Bottom row, higher magnification of cells from the boxed region. Arrows denote tdTomato/nuc.mT-Sapphire double-positive cells that are Olig2 ${ }^{+}$, indicating OPCs derived from dorsal Ascl1 ${ }^{+}$RGCs. Arrowhead denotes a tdTomato/nuc.mT-Sapphire double-positive cell that is Olig2-negative. Scale bar: $10 \mu \mathrm{m}$.

oligodendrocytes are the primary cell type made by $\mathrm{Ascl}_{1}{ }^{+}$dorsal progenitors at this age.

\section{pre-OPCs in developing human brains}

Finally, we wondered if the pre-OPC state identified in mice is conserved in the developing human neocortex. We analyzed data collected from human neocortices at gestational week 18 (Bhaduri et al., 2020), a time developmentally comparable to the end of neurogenesis and beginning of oligodendrogenesis in mice (E16.5-E18.5). Cells collected from various regions of the neocortex were batch corrected before analysis (Fig. 10A). Similar to the mouse datasets, the vast majority of human cells were excitatory neurons (Fig. 10B, Table S8). We identified 2 progenitor clusters, which were re-clustered together for further analysis (Fig. 10B). We found 14 distinct progenitor clusters, the majority of which were RGCs and IPCs (Fig. 10B). We also identified 3 distinct OPC clusters that separated based on maturational stages (Fig. 1oBC). All OPC clusters expressed pan-oligodendrocyte lineage markers $O L I G 1$ and $O L I G 2$, although half of the OPC1 cluster did not express OLIG1 or OLIG2 (Fig. 10D). OPC 3 appeared to be committed oligodendrocyte precursors (COPs) based on expression of genes like BCAS 1 and SIRT2 (Fig. 10C, 
bioRxiv preprint doi: https://doi.org/10.1101/2020.10.18.344515; this version posted October 18,2020 . The copyright holder for this preprint (which was not certified by peer review) is the author/funder, who has granted bioRxiv a license to display the preprint in perpetuity. It is made available under aCC-BY-NC-ND 4.0 International license.
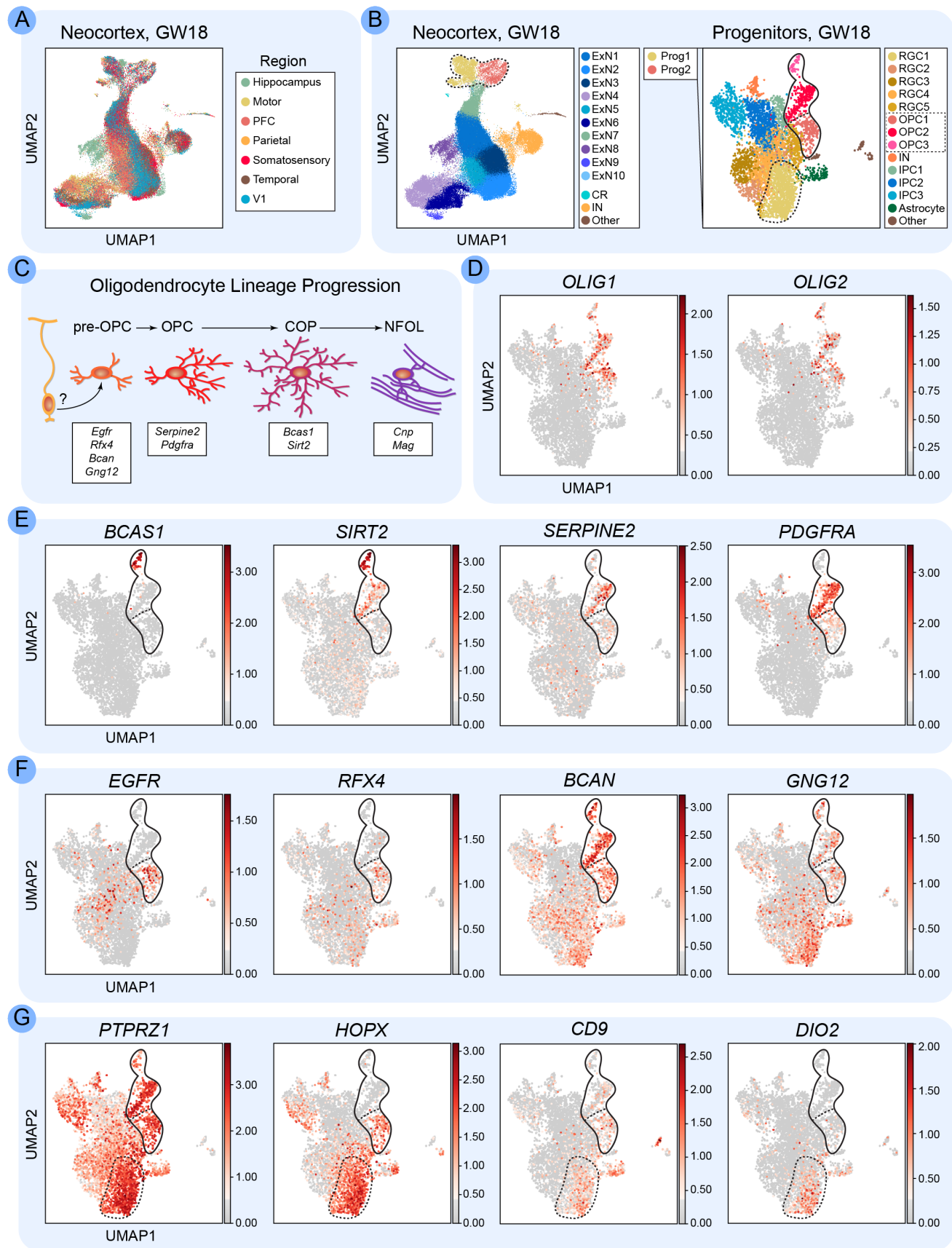

Fig. 10. Identification of pre-OPCs in the human fetal neocortex. (A) Scatterplot of cells after principalcomponent analysis and UMAP visualization of human neocortical cells from gestational week 18 . Several different neocortical regions were sampled and the data were batch corrected accordingly. (B) Left: UMAP visualization of cell clusters identified by Scanpy and annotated by major cell type. "Other" denotes microglia and endothelial cells. Right: resulting UMAP visualization after re-clustering the progenitor and OPC clusters. Fourteen clusters were identified and annotated based on gene expression: radial glial cells (RGC1-5); oligodendrocyte precursor cells (pre-OPC, OPC, COP); interneurons (IN); intermediate progenitor cells (IPC1-3); Astrocytes; Other (microglia and endothelial cells). (C) Schematic representing distinct stages of oligodendrocyte development. Neural progenitor cells give rise to pre-OPCs (characterized by EGFR, RFX4, BCAN and GNG12 expression). pre-OPCs mature into OPCs (characterized by SERPINE2 and PDGFRA), which produce committed precursors (COPs, characterized by BCAS1 and SIRT2) that develop into newly formed oligodendrocytes (NFOL, characterized by $C N P$ and MAG). (D-G) UMAPs of progenitor cells colored by mean expression of: (D) OLIG1 and OLIG2; (E) COP and OPC markers; (F) pre-OPC markers; (G) pre-OPC/oRG-enriched gene PTPRZ1 and oRG markers HOPX, $C D 9$, and DIO2. Some oRG markers were exclusive to the RGC1 cluster (DIO2), whereas others were also expressed in the pre-OPC cluster (PTPRZ1, HOPX, CD9). See also Table S8 for marker genes. 
E). OPC2 was identified as true OPCs based on expression of genes like SERPINE2 and PDGFRA (Fig. $10 \mathrm{C}, \mathrm{E})$. Some of these OPC markers were also weakly expressed in part of the OPC1 cluster (Fig. 10E). Because none of these known oligodendrocyte lineage markers fully covered the OPC 1 cluster, we hypothesized that this cluster might predominantly comprise pre-OPCs. Indeed, several pre-OPC genes identified in mice nicely incorporated the OPC1 cluster (Fig. 10F). Interestingly, none of the pre-OPC genes were exclusive to OPC1; rather, they either spanned the OPC1 and RGC clusters (e.g., EGFR and $\left.R F X_{4}\right)$ or they spanned all OPC clusters and some RGC clusters (e.g., BCAN and GNG12) (Fig. 1oF). Many of these pre-OPC genes were enriched specifically in RGC1 (Fig. $10 \mathrm{~B}, \mathrm{~F}$ ), which were identified as outer radial glial cells (oRGs). oRGs preferentially express PTPRZ1 and HOPX (Pollen et al., 2015), which are both expressed in the OPC1 cluster (Fig. 10G). $P T R P Z_{1}$ was enriched in the RGC1, $\mathrm{OPC}_{1}$, and $\mathrm{OPC}_{2}$ clusters, while HOPX was even further restricted to predominantly the RGC1 and OPC1 clusters (Fig. 10G). We also found CD9 expression to be relatively restricted to the RGC1 and OPC1 clusters (Fig. 10G). oRGs also express genes associated more with astrocytes later in development, like $\mathrm{DIO}_{2}$ (Pollen et al., 2015), which we found in both the RGC1 and astrocyte clusters, but not in the OPC clusters (Fig. 10G). Taken together, our analysis of the developing human neocortex identified a potential pre-OPC stage in which progenitors express low levels of some OPC markers and several pre-OPC markers identified in mice, indicating conservation of certain aspects of the pre-OPC state from mice to humans.

\section{DISCUSSION}

Neural progenitors in the mammalian dorsal forebrain generate diverse cell types for the neocortex. An initial period of excitatory neuron production is followed by a later phase in which the progenitor pool switches to making oligodendrocytes, astrocytes and olfactory bulb interneurons. Several studies have implicated Shh signaling in this transition, but the molecular mechanisms downstream of Shh are not fully understood. In this study, we analyzed scRNAseq datasets to uncover transcriptional changes as progenitors transition to making OPCs in response to Shh. We found that 1) Shh signaling promotes a preOPC transcriptional state that emerges in progenitors prior to OPC appearance; 2) this pre-OPC state is characterized by enrichment of a gene regulatory network involving Ascl1 that has been shown to oppose an excitatory neuron transcriptional network; 3) the majority of cells generated by $\mathrm{Ascl}_{1}^{+}$ progenitors in the late embryonic dorsal forebrain belong to the oligodendrocyte lineage. Together with previous work, these data lead us to propose a working model in which increased Shh signaling late in embryonic corticogenesis influences the balance between opposing transcriptional pathways to favor oligodendrogenesis over excitatory neuron fates (Fig. 11).

Multiple Temporal Roles for Shh Signaling in Cell Fate Specification

Throughout the CNS, Shh signaling is critical for setting up dorsal-ventral patterning of progenitor domains and for subsequently modulating diverse fates of their differentiated progeny, including oligodendrocytes (Andrews et al., 2019; Yabut and Pleasure, 2018). Recent studies have started to elucidate how levels and timing of Shh signaling are tightly regulated to control the identities of both progenitors and their progeny. Our lab uncovered a critical timing mechanism for Shh-mediated oligodendrogenesis in the developing neocortex (Winkler et al., 2018). Early in corticogenesis Shh signaling in the dorsal forebrain is low, due to weak expression of the ligand and high expression of the negative regulator, Suppressor of Fused (Sufu). This allows for proper dorsal-ventral patterning of forebrain progenitors. Later in corticogenesis, however, Shh signaling increases in the dorsal forebrain and is required for initiation of oligodendrogenesis. This is accomplished partly by decreased expression of Sufu (Yabut et al., 2015; Yabut et al., 2016), as well as increased Shh ligand brought in by interneurons migrating from the ventral forebrain (Winkler et al., 2018). In this way, tight control over the levels and timing of Shh signals confers both spatial patterning early in development and the later switch from neurogenesis to oligodendrogenesis.

Interestingly, recent studies together with our data presented here suggest that the molecular mechanisms involved in these dual roles might be very similar. Genetic ablation of the Shh repressor, Sufu, in 

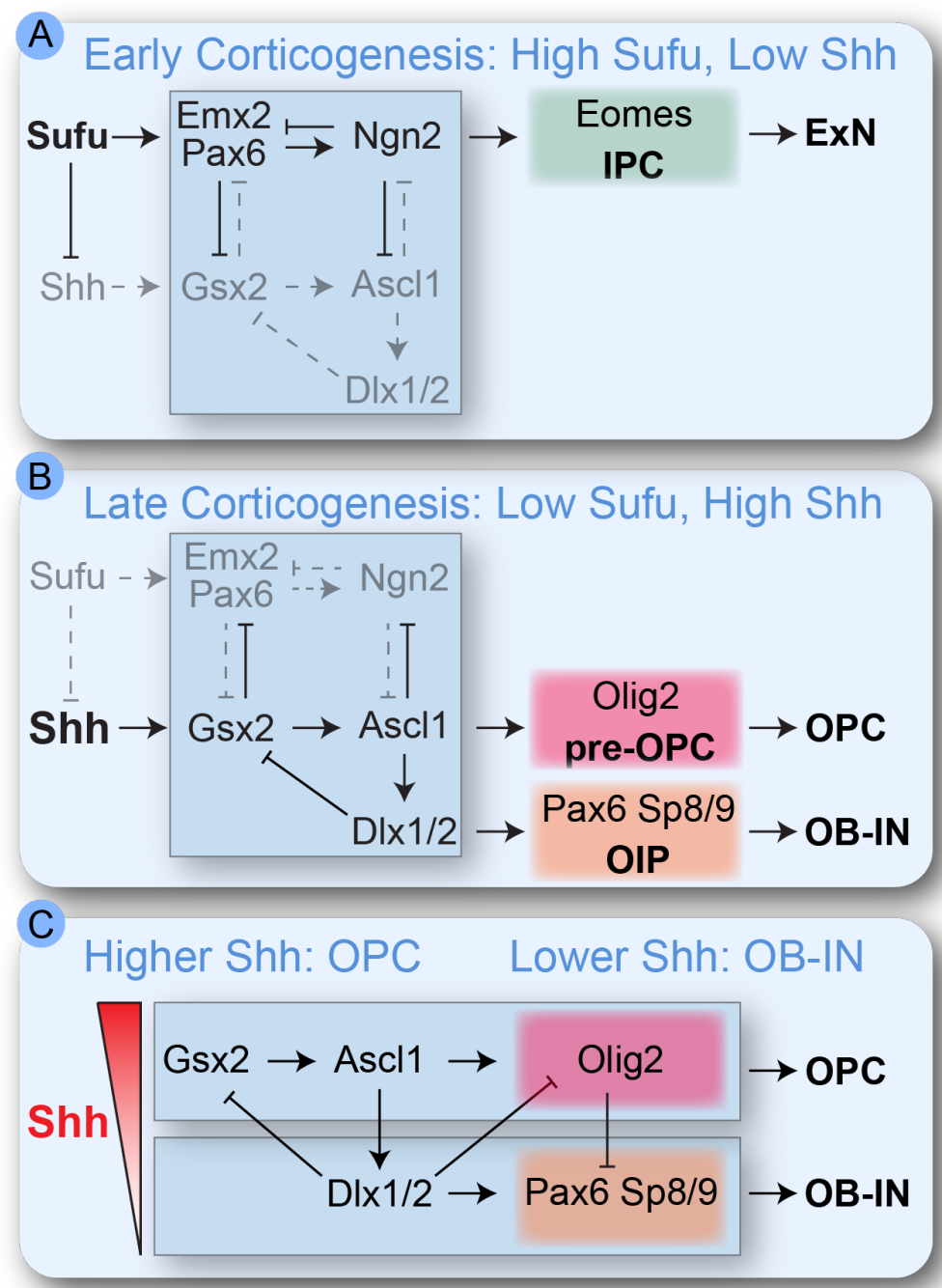

Fig. 11. Working model for preOPC fate specification by Shh signaling.

(A) Early in corticogenesis Shh signaling in the dorsal forebrain is low, due to low levels of Shh ligand and high expression of Sufu. This allows the Emx2/Pax6 $\rightarrow$ Neurog 2 $\rightarrow$ Eomes pathway to repress ventral identity genes and drive specification and neurogenesis of excitatory neurons. (B) Later in corticogenesis Shh ligand increases in the dorsal forebrain while Sufu expression decreases. This promotes transcription of the ventral Gsx2 $\rightarrow$ Ascl1 $\rightarrow$ Dlx1/2 \& Olig2 pathways, which repress dorsal excitatory neuron identity genes and drive specification of OPCs and OB-INs. (C) Levels of Shh signaling are also important for the fate decision between the OPC and OBIN lineages, where the highest levels of Shh signaling promote oligodendrogenesis and lower Shh signaling promotes OB-IN generation through another crossrepressive transcriptional network. the early dorsal forebrain leads to overactivation of Shh-mediated transcription, resulting in patterning defects in dorsal progenitors (Yabut et al., 2015; Yabut et al., 2016; Yabut et al., 2020). The mis-specified dorsal progenitors ectopically express ventral identity genes, including Gsx1/2, Dlx1/2, Olig2 and Ascl1, and downregulate dorsal identity genes like $E m x_{1} / 2$ and Pax6 (Yabut et al., 2015; Yabut et al., 2020). Thus, precocious activation of Shh signaling in dorsal progenitors activates a ventral transcriptional program. Our scRNA-seq analysis revealed a similar transcriptional network at later stages during oligodendrocyte lineage specification, in which Gsx2, $D x_{1} / 2$ and Ascl 1 were all enriched in ShhPCs. Therefore, we hypothesize that Shh signaling employs the same ventral transcriptional pathway in both early progenitor patterning and later OPC specification.
Cross-repressive Gene Regulatory Networks for Neurogenesis and Oligodendrogenesis

A number of transcription factors work together in a network that confers dorsal identity and promotes specification of glutamatergic excitatory neurons for the neocortex. Emx2, Lhx2 and Pax6 are expressed in dorsal progenitors, where they are critical for establishing patterns of regional and areal identities and for maintaining progenitor proliferation (Hevner, 2006). Pax6 is also important for activating transcription of proneural genes Neurog 1 and Neurog2 (Scardigli, 2003). Pax6 and Neurog $1 / 2$ are required for proper specification of a glutamatergic excitatory neuron identity (Schuurmans et al., 2004). Production of neocortical excitatory neurons most commonly proceeds through intermediate progenitor cells (Kowalczyk et al., 2009) that express the transcription factor Tbr2 (encoded by Eomes). 
Neurog2 regulates the transition from RGCs to IPCs by downregulating Pax6 and initiating Tbr2 expression (Kovach et al., 2013). Thus, a transcriptional pathway involving Emx2/Pax6 $\rightarrow$ Neurogi/2 $\rightarrow$ Eomes is central to the fate specification and genesis of glutamatergic excitatory neurons in the neocortex (Fig. 11A).

This dorsal transcriptional pathway participates in a cross-repressive network with an opposing pathway that confers ventral progenitor identities and GABAergic interneuron cell fates (Fig. 11A). In Emx2 I- and $\mathrm{Pax6}^{-1-}$ mutant forebrains, dorsal progenitors ectopically express transcription factors in the ventral

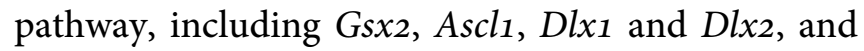
generate GABAergic inhibitory interneurons instead of glutamatergic excitatory neurons (Kroll and O'Leary, 2005). Emx2 and Pax6 can repress transcription of Gsx2 (Cocas et al., 2011; Coutinho et al., 2011; Desmaris et al., 2018; Sun et al., 2015), and Pax6 and Neurog2 can similarly repress Ascl1 expression (Gowan et al., 2001; Roybon et al., 2010; Sun et al., 2015). Conversely, knockout of Gsx2 leads to ectopic expression of Emx2, Pax6 and Neurog2 in the ventral forebrain (Chapman et al., 2013; Corbin et al., 2000; Toresson et al., 2000; Yun et al., 2001), indicating that the ventral transcriptional network can also repress the dorsal pathway at multiple points.

Our analyses indicate that the ventral Gsx2 $\rightarrow$ Ascl $_{1} \rightarrow$ $D l x_{1} / 2$ transcriptional pathway is enriched in ShhPCs. This is consistent with some ShhPCs being committed to the OB-IN lineage (Zhang et al., 2020), as this pathway is important for generating OB-INs (Guo et al., 2019; Lindtner et al., 2019). In addition to its roles in neurogenesis, Ascl1 genetically interacts with Olig2 and is critical for normal production of oligodendrocytes in the spinal cord and ventral forebrain (Nakatani et al., 2013; Parras et al., 2007; Sugimori et al., 2007). Olig2 can also repress Pax6, and both Pax6 and Tbr2 expression are reduced when Olig2 is overexpressed in the neocortex via electroporation ( $\mathrm{Lim}$ et al., 2019). Based on these previous studies and the analyses presented here, we propose a model for how Shh signaling controls the switch from excitatory neurogenesis to production of OPCs and OB-INs (Fig. 11). Early in corticogenesis (Fig. $11 \mathrm{~A}$ ), Shh ligand expression is low dorsally, whereas Sufu expression is high in dorsal RGCs. This low Shh signaling state allows the Emx2/Pax6 $\rightarrow$ Neurog $1 / 2 \rightarrow$ Eomes pathway to repress ventral identity genes and drive specification and neurogenesis of excitatory neurons (Fig. 11A). Once the majority of excitatory neurons have been generated (Fig. 11B), Shh ligand increases in the dorsal forebrain while Sufu expression decreases, leading to increased Shh signaling in dorsal progenitors. High Shh signaling promotes transcription of the ventral Gsx $\rightarrow A s c l_{1} \rightarrow D l x_{1} / 2$ \& Olig2 pathways, which repress excitatory neuron identity genes and drive specification of OPCs and OB-INs (Fig. 11B).

A similar cross-repressive mechanism may be involved in the next fate decision between OPCs and OB-INs (Fig. ${ }_{11} \mathrm{C}$ ). The ShhPC cluster was further split into pre-OPCs and OB-IN precursors (OIPs). Pre-OPCs maintained higher levels of Gsx2, Ascl 1 and Olig2 along pseudodevelopmental time, whereas OIPs increased their expression of Dlx1/2, Pax6 and Sp8/9. Ascl 1 can activate transcription of both Olig2 (Vue et al., 2020) and $D l x_{1} / 2$ (Castro et al., 2011). In the ventral forebrain, Dlx1/2 negatively regulate Olig2dependent OPC formation, whereas Ascl1 promotes OPC formation by restricting the number of $\mathrm{Dlx} 1 / 2^{+}$ cells (Petryniak et al., 2007). In the developing chick forebrain, Dlx1/2 are necessary and sufficient to repress oligodendrocyte specification via transcriptional inhibition of Olig2 (Jiang et al., 2020). Dlx1/2 also repress Gsx2 (Guo et al., 2019) and activate $S p 8$ and $S p 9$ expression (Lindtner et al., 2019), which are essential for OB-IN maturation (Guo et al., 2019; Li et al., 2018). Pax6 is also required to generate a subset of OB-INs (Kohwi et al., 2005), and we found that Pax6 levels are initially decreased in ShhPCs before increasing again specifically in the OB-IN lineage. Repression of Pax6 by Olig2 (Jang and Goldman, 2011) might therefore be important for maintaining an OPC identity over that of OB-INs. Olig2 also activates Olig1 expression and Olig1 directly represses $D l_{x 1 / 2}$ to inhibit interneuron production (Silbereis et al., 2014). Together, these studies raise the possibility that the relative levels of these cross-repressive transcription factors determine final fate commitment to either OPC or OB-IN lineages (Fig. $11 \mathrm{C}$ ). Interestingly, we found that transcripts involved in Shh signaling were higher in the OPC lineage compared to the OB-IN lineage, 
suggesting that the levels of Shh may again be important for determining this binary fate choice. We propose that ShhPCs experiencing the highest levels of Shh signaling commit to the OPC lineage, whereas those with lower Shh signaling are fated to become OB-INs (Fig. 11C).

\section{Role of Ascl1 in Specifying Oligodendrocyte Fate} Downstream of Shh

Our analyses revealed $A s c l 1$ as a marker of ShhPCs, consistent with experimental data that Shh signaling promotes Ascl1 expression (Voronova et al., 2011; Wang et al., 2007). Although Ascl1 is most highly expressed in ventral forebrain progenitors, we and others have shown that Ascl1 is expressed at low levels in dorsal progenitors (Britz et al., 2006; Fode et al., 2000; Winkler et al., 2018). Ascl1 is an early marker of the oligodendrocyte lineage in other CNS regions (Nakatani et al., 2013; Vue et al., 2014), and we confirm that Ascl 1 is expressed in dorsal RGCs prior to expression of Olig2. We also previously demonstrated that dorsal Ascl1 expression requires Shh signaling (Winkler et al., 2018). Together, these data indicate that $A s c l 1$ is one of the first genes upregulated in the oligodendrocyte lineage in response to Shh. Previous lineage-tracing studies indicated that neocortical cells derived from the Ascl 1 lineage were oligodendrocytes and ventrally-derived interneurons, but not astrocytes or excitatory neurons (Kim et al., 2008; Kim et al., 2011). Here, we combined directional in utero electroporation with short-term Ascl1-CreERT2 lineage tracing to show that the majority of cells in the dorsal Ascli lineage were OPCs. The identities of the $\sim 30 \%$ that are not OPCs remain to be determined, but transcriptomics and previous lineage analyses indicate that many are likely OB-IN neuroblasts.

Interestingly, a recent preprint found that E12.5 dorsal progenitors fall into one of four categories based on their expression of Ascl1 and Neurog2 (Han et al., 2020). The majority of progenitors were doublenegative or $\mathrm{Neurog}_{2}{ }^{+}$, but a small proportion expressed Ascl1 or Ascl1 and Neurog 2 together. They found by scRNA-seq that the $\mathrm{Ascl}^{+}$cells were enriched for oligodendrocyte and interneuron genes and were biased towards generating OPCs when cultured in vitro. Their in silico modeling indicated that "knocking out" Ascl1 perturbed a major gene regulatory network in $\mathrm{Ascl}^{+}$cells. These results are compatible with our in vivo lineage tracing and further support a critical role for Ascl1 in specifying the oligodendrocyte lineage in the dorsal forebrain.

Another interesting finding from Han et al. is that the Ascl1;Neurog 2 double-negative and the $\mathrm{Ascl}_{1}{ }^{+}$ progenitor subsets were the most RGC-like, whereas the Neurog $2^{+}$and double-positive cells expressed higher levels of IPC and neuronal genes. We observed a similar pattern in our analyses, in which Ascl $^{+}$ ShhPCs expressed several RGC markers and exhibited weaker RNA velocities compared to more differentiated cells. This connection between preOPCs and RGCs was even stronger in the human dataset, in which the pre-OPC cluster shared several markers with outer radial glia (oRG). Indeed, a recent study showed that oRGs are lineally related to preOPCs in the human neocortex (Huang et al., 2020). It will be interesting to test in the future whether this relationship between pre-OPCs and RGCs has any functional connection to the long-term proliferative capacity of OPCs, and whether Shh signaling is involved.

\section{Conclusion}

We propose a model in which Shh signaling promotes an oligodendrocyte fate by influencing the balance between ventral and dorsal identities in a crossrepressive gene regulatory network. Future studies should shed light on the specific functions of individual transcription factors in this process, in particular the roles of Ascl1. It will also be interesting to determine whether a similar mechanism is at work during OPC production in other contexts, for example in the developing spinal cord or in the postnatal brain. Further investigations into oligodendrocyte development in the embryonic brain may also reveal insights into OPC responses to disease or injury in the postnatal brain, and potential strategies for treatment.

\section{MATERIALS AND METHODS}

Computational Analysis

\section{Software}

For all analyses we used a combination of Python (version 3.7.4) and $\mathrm{R}$ (version 3.6.2) packages and 
integrated $\mathrm{R}$ into Jupyter Notebook using the rpy2 interface (version 3.0.5) and anndata2ri (version 1.0.4) (https://github.com/theislab/anndata2ri), which automatically converts from an AnnData object to a SingleCellExperiment object.

\section{Samples}

\section{Shh-IUE Mouse Cortex}

FASTQ files were downloaded from the National Center for Biotechnology Information BioProjects Gene Expression Omnibus (GEO: GSE140817; ShhIUE samples) (Zhang et al., 2020) and processed using Cell Ranger (version 2.1.1) with mm1o genome assembly to generate unique molecular identifier (UMI) gene count matrices. An aggregated matrix was also generated by downsampling of the mapped reads in each sample to the same depth as the sample with the lowest read count using cellranger aggr. The aggregated matrix was processed using Scanpy (version 1.4.6) (Wolf et al., 2018) to perform initial quality control filtering, normalization, and clustering.

\section{WT Mouse Neocortex}

For embryonic ages E11.5, E13.5, and E15.5 we downloaded provided tab-delimited text files containing raw digital gene expression for all cells (GEO: GSE107122) (Yuzwa et al., 2017). For embryonic ages E12.5, E16.5, and E18.0 we downloaded provided developmental data as a single file (dev_all.loom) from the Mouse Brain Atlas website compiled by the Linnarsson lab (mousebrain.org). We then extracted the developmental timepoints of interest as separate $h_{5} \mathrm{ad}$ files. Both the tab-delimited text files and h5ad files were read in and converted to AnnData objects for easy integration into our Scanpy pipeline for preprocessing and downstream analysis.

\section{Human Neocortex}

We downloaded the raw expression matrix for primary human cortex samples directly from the provided UCSC cell browser website (https://cells.ucsc.edu/?ds=organoidreportcard), as they were not yet available through the database of Genotypes and Phenotypes $(\mathrm{dbGaP})$ at the time of analysis (GEO: GSE132672) (Bhaduri et al., 2020). We processed the raw expression matrix in $\mathrm{R}$ to selectively isolate samples from gestational week 18 (GW18). We used Seurat (version 3.1.5) to create a SeuratObject of the GW1 8 dataset, which we then converted to an AnnData object that we could easily integrate into our Scanpy pipeline for pre-processing and downstream analysis.

\section{Pre-processing}

Pre-processing and downstream analysis on the data sets was conducted following the steps outlined by Luecken and Theis (2019). For all data sets, genes were excluded if they were detectable in fewer than 5 cells. For Shh-IUE data, cells were removed if there were fewer than 1000 genes detected, a UMI count less than 2500, a UMI count more than 20,000, or if the proportion of UMIs mapped to mitochondrial genes was greater than $15 \%$. We also excluded cells if the proportion of UMIs mapped to ribosomal genes was greater than $18 \%$ on a second pass through of the data, as we noticed that these cells represented stressed and/or dying cells. This resulted in 8154 cells (from 9080 total cells) after filtering. For E11.5 WT data, cells were removed if there were fewer than 500 genes detected, a UMI count less than 700, a UMI count more than 10,000 , or if the proportion of UMIs mapped to mitochondrial genes was greater than $5 \%$, resulting in 1837 cells (from 2000 total cells) after filtering. For E13.5WT data, cells were removed if there were fewer than 500 genes detected, a UMI count less than 500, a UMI count more than 10,000, or if the proportion of UMIs mapped to mitochondrial genes was greater than $20 \%$, resulting in 1729 cells (from 2000 total cells) after filtering. For E15.5WT data, cells were removed if there were fewer than 500 genes detected, a UMI count less than 750, a UMI count more than 20,000, or if the proportion of UMIs mapped to mitochondrial genes was greater than $15 \%$, resulting in 4725 cells (from 5000 total cells) after filtering. For E12.5WT data, cells were removed if there were fewer than 1000 genes detected, a UMI count less than 2000, a UMI count more than 10,000 , or if the proportion of UMIs mapped to mitochondrial genes was greater than $2 \%$, resulting in 2378 cells (from 2601 total cells) after filtering. For E16.5 WT data, cells were removed if there were fewer than 1000 genes detected, a UMI count less than 1000 , a UMI count more than 15,000 , or if the proportion of UMIs mapped to mitochondrial genes was greater than $20 \%$, resulting in 2871 cells (from 3185 total 
cells) after filtering. For E18.o WT data, cells were removed if there were fewer than 1000 genes detected, a UMI count less than 1000, a UMI count more than 10,000 , or if the proportion of UMIs mapped to mitochondrial genes was greater than $20 \%$, resulting in 4045 cells (from 4859 total cells) after filtering. For $\underline{G W 18}$ data, cells were removed if there were fewer than 500 genes detected, a UMI count less than 1000, a UMI count more than 12,000 , or if the proportion of UMIs mapped to mitochondrial genes was greater than $10 \%$, resulting in 73623 cells (from 78453 total cells) after filtering.

Following filtering, the UMI counts for all data sets were normalized via scran normalization (version 1.14.6) and log-transformed with an offset of 1. For the $\underline{G W 18}$ data, following normalization we used ComBat batch correction (Johnson et al., 2007), as cells were collected from various regions across the neocortex and were clustering based on region. To reduce the dimensionality of each dataset the top 4000 highly variable genes were extracted for further processing. Dimensions of each dataset were further reduced by principal component analysis (using the top 50 principal components) and visualized using Uniform Manifold Approximation and Projection (UMAP). Finally, we used a gene list from Macosko et al. (Macosko et al., 2015) to score the cell cycle effect in each data set and classify cells by cell cycle phase.

\section{Cluster identification and annotation}

Graph-based clustering was performed on the highly variable gene data for each data set, dimensionality reduced by Principal Component Analysis (PCA), and embedded into K-Nearest Neighbors (KNN) graph using the Louvain algorithm with a resolution of 1.o. Clusters were annotated using a combination of marker genes generated by differential expression testing (using the Wilcoxon rank-sum test) and by visualizing expression of known marker genes of various cortical cell types (see Table $S_{1}$ for mouse genes, Table S8 for human genes). We analyzed the expression of both individual genes as well as marker gene sets that we compiled based on differential expression testing and published literature (Tables $\mathrm{S}_{1}$ and S8). After cluster annotations, we repeated the clustering as described on a subset of cells that included all progenitor cell types (and immature olfactory bulb interneurons (OB-IN) in the Shh-IUE data set). Here we regressed out cell cycle effects to avoid progenitors clustering based on cell-cycle stage. For the Shh-IUE data, in order to further subcluster the Shh-responding progenitor cell (ShhPC) cluster we performed Louvain clustering on just the ShhPC cluster using a resolution of 1.0 .

\section{Functional enrichment analysis}

We used the g:Profiler web server (https://biit.cs.ut.ee/gprofiler/gost) to perform functional enrichment analysis on the ShhPC1 and $\mathrm{ShhPC}_{3}$ clusters. Lists of differentially expressed genes in ShhPC1 versus rest (Table $\mathrm{S}_{4}$ ) and ShhPC2 versus rest (Table S6) were generated using built-in features of Scanpy (using the Wilcoxon rank-sum test). We used g:Profiler to map these gene lists to known functional information sources and detect statistically significantly enriched terms (Tables $\mathrm{S}_{5}$ and $\left.\mathrm{S}_{7}\right)$.

\section{Trajectory analysis}

The following downstream analysis was only performed in the Shh-IUE data set. Cell lineage trajectory within the progenitor subset was analyzed using Slingshot (version 1.5.1) (Street et al., 2018). Dimensionality was reduced by PCA, and we used an unbiased approach for lineage construction by only specifying a start cluster (radial glial cells, or RGCs, the most stem-like progenitor cluster). This predicted two distinct lineage trajectories (an oligodendrocyte lineage and OB-IN lineage) which we visualized using Slingshot visualization tools.

We also did RNA velocity analysis as a second, independent method of trajectory analysis. We used the Python implementation of velocyto (version o.17.13) (La Manno et al., 2018), using the basic 'run' subcommand with our Cell Ranger output and mm1o genome assembly. We also opted to mask expressed repetitive elements using the mm1o expressed repeat annotation file downloaded from the UCSC genome browser. We analyzed the output loom file using scVelo (version 0.2.2) (Bergen et al., 2020), and merged the loom file with our Scanpy-processed and -annotated progenitor subset. The resulting abundance of spliced to unspliced RNA was 0.73:0.27. We set the minimum number of counts (both unspliced and spliced) required for a gene to 1 and 
filtered out 1446 genes. We used default arguments in order to compute moments for velocity estimation.

\section{Trajectory-based differential expression analysis}

We used tradeSeq (version 1.1.16) (Van den Berge et al., 2020) to infer differential gene expression between the lineages predicted by our Slingshot analysis. We used $\mathrm{k}=8$ knots to fit the tradeSeq negative binomial generalized additive model for each gene in the Slingshot dataset and estimated one smoother for each of our two lineages (default parameters). We used built-in tools provided by tradeSeq to generate lists of genes that were differentially expressed between the oligodendrocyte and OB-IN lineages between knots 2 and 5 (Table $S_{3}$ ), as this is when the lineages first began to bifurcate. Smoothers were visualized using built-in tools.

\section{Experimental Methods}

Mice

The following mice were obtained from The Jackson Laboratory: B6 ( $C_{57} \mathrm{BL} / 6 \mathrm{~J}$; stock no. o0o664); Ai9

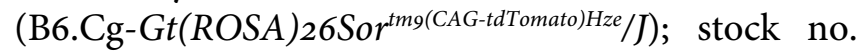
007909); Ascl1-CreERT2 (Ascl ${ }^{\text {tm1.1(Cre/ERT2)Jejo } / J) ; ~ s t o c k ~}$

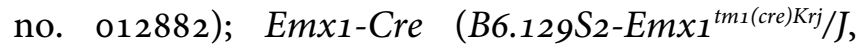
stock no. 005628); R26-NZG [FVB.CgGt(ROSA)26Sor ${ }^{\operatorname{tm} 1(C A G-l a c Z,-E G F P) G l h} / J$, stock no. 012429]. Emx1- ${ }^{\mathrm{Cre} / \mathrm{Cre}}$ homozygous mice were crossed with R26NZG/NZG homozygous mice to generate double heterozygous embryos for analysis. Ascl1 $1^{+/ C_{r e E R T 2}}$ heterozygous males were crossed to $A i 9^{\mathrm{f} / f l}$ homozygous females to generate pregnant dams for electroporation and tamoxifen injection. Animals were maintained according to the guidelines from the Institutional Animal Care and Use Committee of the University of Colorado - Anschutz Medical Campus. Sex of experimental embryos was not determined in our experiments.

\section{Tissue preparation}

Embryonic brains were fixed in $4 \%$ paraformaldehyde (PFA) for $1 \mathrm{~h}$ at room temperature (RT). Postnatal day o (Po) brains were fixed in $4 \%$ PFA overnight at 4 degrees C. For immunohistochemistry, brains were sectioned coronally at $50-100 \mu \mathrm{m}$ with a vibrating microtome. For RNAscope ${ }^{\oplus}$ (ACD) mRNA in situ hybridization, brains were cryoprotected in $30 \%$ sucrose at 4 degrees $\mathrm{C}$ overnight and sectioned on a cryostat at $12 \mu \mathrm{m}$.

\section{Immunohistochemistry}

Free-floating vibratome sections were blocked with $10 \%$ donkey serum and $0.2 \%$ Triton-X in $1 \mathrm{X}$ PBS for $2 \mathrm{~h}$ at RT. After $2 \mathrm{~h}$, the blocking solution was removed and sections were incubated with primary antibodies in $10 \%$ donkey serum in $1 \mathrm{X}$ PBS overnight at 4 degrees $\mathrm{C}$, and then washed at RT with $1 \mathrm{X}$ PBS three times for $5 \mathrm{~min}$ each. After washing, sections were incubated with secondary antibodies in $10 \%$ donkey serum in $1 \mathrm{X}$ PBS for $1 \mathrm{~h}$ at RT, and then washed again with 1 X PBS three times for 5 min each. Sections were mounted on slides with ProLong Diamond Antifade Mountant (Invitrogen $\mathrm{P}_{3} 6961$ ). Images were captured using a LSM9oo Zeiss laserscanning confocal microscope in Airyscan 2 Multiplex $4 \mathrm{Y}$ mode at 20x (PlanApo 20x/o.8 M27, Zeiss 420650-9902-00o) or 40x (C-Apo 40x/1.20 W Corr M27, Zeiss 421767-9971-00o) magnification. Antibodies used for immunostaining wild-type B6 brains were: rabbit anti-Ascl1 (1:1000; Abcam [EPR19840] ab211327), mouse anti-Olig2 (1:500; Millipore MABN50; RRID:AB_10807410), chicken anti- $\beta$-gal (1:2000; Abcam ab9361; RRID:AB_307210). Rabbit anti-Olig2 (1:500; Millipore AB9610; RRID:AB_570666) was used for immunostaining electroporated brain sections. Donkey secondary antibodies conjugated to Alexa Fluor 488, Rhodamine Red-X, or Alexa Fluor 647 were purchased from Jackson ImmunoResearch and used at 1:500.

\section{RNAscope ${ }^{\oplus}$ mRA in situ hybridization} mRNA in situ hybridization was performed using the RNAscope $^{\circledast}$ Multiplex Fluorescent Reagent Kit v2 (Advanced Cell Diagnostics) with an Ascl1 probe (RNAscope ${ }^{\varpi}$ Probe - Mm-Ascl1-C2; Advanced Cell Diagnostics, catalog no. 313291-C2) and an Emx1 probe (RNAscope ${ }^{\oplus}$ Probe - Mm-Emx1; Advanced Cell Diagnostics, catalog no. 312261). Signals were visualized with Opal 520 (Akoyo Biosciences FP1487001KT) and Opal 570 (Akoyo Biosciences $\mathrm{FP} 1488001 \mathrm{KT})$. The assay was performed in accordance with the manufacturer's instructions, except that slides were steamed in target retrieval buffer lying flat horizontally instead of vertically, to 
prevent sections from sliding. Sections were counterstained with DAPI to label nuclei.

\section{In Utero Electroporation and Tamoxifen Injection}

A piggyBac (PB) transposase system was used for in utero electroporation experiments to permit stable integration of the reporter plasmid into electroporated progenitors (Silbereis et al., 2014). pPB-nuc.mT-Sapphirie was generated by removing the Lox-STOP-Lox cassette from pPB-STOPnuc.mT-Sapphire (García-Moreno et al., 2014) by incubating $0.25 \mu \mathrm{l}$ of plasmid with $1 \mu \mathrm{l}$ of recombinant Cre recombinase (New England Biolabs Mo298S) according to manufacturer's instructions. In utero electroporations were performed as described (Winkler et al., 2018). Briefly, timed pregnant mice (E15.5) were anesthetized and their uterine horns exposed. $1 \mu$ of endotoxin-free plasmid DNA was injected into the embryos' lateral ventricles at the following concentrations: pPB-nuc.mT-Sapphire 1 $\mathrm{mg} / \mathrm{mL}, \mathrm{CMV}-\mathrm{mPB}$ 0.3 mg/mL (Winkler et al., 2018). 5 electrical pulses separated by $950 \mathrm{~ms}$ were applied at $45 \mathrm{~V}$. Tamoxifen injections of the pregnant dams were performed immediately after the electroporated embryos were returned to the abdominal cavity and the dams were sutured. Tamoxifen (Sigma-Aldrich $\mathrm{T}_{5648)}$ was dissolved in corn oil (Sigma-Aldrich C8267) at (10 $\mathrm{mg} / \mathrm{ml})$ and administered by intraperitoneal injection of $2 \mathrm{mg}$. Embryos were allowed to develop in utero for the indicated time. For quantification of electroporated cells that were Olig2 ${ }^{+}$, all nuc.mT-Sapphire;tdTomato double-positive electroporated cells in each entire section were analyzed for Olig2 expression by IHC with rabbit antiOlig2.

\section{Acknowledgements}

We thank members of the Pediatrics Department Section of Developmental Biology and the RNA Biosciences Informatics Fellows for helpful discussions.

\section{Competing interests}

The authors declare no competing or financial interests.

\section{Author contributions}

Conceptualization: C.C.W., S.J.F.; Methodology: C.C.W., L.N.T., E.P.M., F.G.-M., S.J.F.; Formal analysis: C.C.W.; Investigation: C.C.W., L.N.T., E.P.M., S.J.F.; Resources: F.G.-M.; Data curation: C.C.W.; Writing - original draft preparation: C.C.W., S.J.F.; Writing - review and editing: L.N.T.; Visualization: C.C.W., L.N.T., S.J.F.; Supervision: C.C.W., S.J.F.; Project administration: C.C.W., S.J.F.; Funding acquisition: C.C.W., S.J.F.

\section{Funding}

This work was supported by National Institute of Neurological Disorders and Stroke (NINDS) / National Institutes of Health (NIH) grant Ro1NS109239. C.C.W. was supported by an RNA Biosciences Informatics Fellowship and the Postdoctoral Training Program in Developmental Biology and Regenerative Medicine at the University of Colorado - Anschutz Medical Campus. L.N.T. was supported by a National Institutes of Health training grant to the Molecular Biology PhD Program at the University of Colorado - Anschutz Medical Campus (T32-GM136444).

Data availability

Jupyter Notebook workflows available upon request.

\section{REFERENCES}

Andrews, M. G., Kong, J., Novitch, B. G. and Butler, S. J. (2019). New perspectives on the mechanisms establishing the dorsal-ventral axis of the spinal cord. In Current Topics in Developmental Biology, pp. 417-450. Elsevier.

Battiste, J., Helms, A. W., Kim, E. J., Savage, T. K., Lagace, D. C., Mandyam, C. D., Eisch, A. J., Miyoshi, G. and Johnson, J. E. (2007). Ascl1 defines sequentially generated lineage-restricted neuronal and oligodendrocyte precursor cells in the spinal cord. Development 134, 285-293.

Bergen, V., Lange, M., Peidli, S., Wolf, F. A. and Theis, F. J. (2020). Generalizing RNA velocity to transient cell states through dynamical modeling. Nat. Biotechnol.

Bhaduri, A., Andrews, M. G., Mancia Leon, W., Jung, D., Shin, D., Allen, D., Jung, D., Schmunk, G., Haeussler, M., Salma, J., et al. (2020). Cell stress in cortical organoids impairs molecular subtype specification. Nature 578, 142-148.

Britz, O., Mattar, P., Nguyen, L., Langevin, L.-M., Zimmer, C., Alam, S., Guillemot, F. and Schuurmans, C. (2006). A Role for Proneural Genes in the Maturation of Cortical Progenitor Cells. Cereb. Cortex 16, i138i151. 
Castro, D. S., Martynoga, B., Parras, C., Ramesh, V., Pacary, E., Johnston, C., Drechsel, D., Lebel-Potter, M., Garcia, L. G., Hunt, C., et al. (2011). A novel function of the proneural factor Ascl1 in progenitor proliferation identified by genome-wide characterization of its targets. Genes Dev. 25, 930-945.

Chapman, H., Waclaw, R. R., Pei, Z., Nakafuku, M. and Campbell, K. (2013). The homeobox gene Gsx2 controls the timing of oligodendroglial fate specification in mouse lateral ganglionic eminence progenitors. Development 140, 2289-2298.

Cocas, L. A., Georgala, P. A., Mangin, J.-M., Clegg, J. M., Kessaris, N., Haydar, T. F., Gallo, V., Price, D. J. and Corbin, J. G. (2011). Pax6 Is Required at the Telencephalic Pallial-Subpallial Boundary for the Generation of Neuronal Diversity in the Postnatal Limbic System. J. Neurosci. 31, 5313-5324.

Corbin, J. G., Gaiano, N., Machold, R. P., Langston, A. and Fishell, G. (2000). The Gsh2 homeodomain gene controls multiple aspects of telencephalic development. Dev. Camb. Engl. 127, 5007-5020.

Coutinho, P., Pavlou, S., Bhatia, S., Chalmers, K. J., Kleinjan, D. A. and van Heyningen, V. (2011). Discovery and assessment of conserved Pax6 target genes and enhancers. Genome Res. 21, 1349-1359.

Desmaris, E., Keruzore, M., Saulnier, A., Ratié, L., Assimacopoulos, S., De Clercq, S., Nan, X., Roychoudhury, K., Qin, S., Kricha, S., et al. (2018). $\mathrm{DMRT}_{5}, \mathrm{DMRT}_{3}$, and EMX2 Cooperatively Repress Gsx2 at the Pallium-Subpallium Boundary to Maintain Cortical Identity in Dorsal Telencephalic Progenitors. J. Neurosci. 38, 9105-9121.

Falcão, A. M., van Bruggen, D., Marques, S., Meijer, M., Jäkel, S., Agirre, E., Samudyata, Floriddia, E. M., Vanichkina, D. P., ffrench-Constant, C., et al. (2018). Disease-specific oligodendrocyte lineage cells arise in multiple sclerosis. Nat. Med. 24, 1837-1844.

Fode, C., Ma, Q., Casarosa, S., Ang, S. L., Anderson, D. J. and Guillemot, F. (2000). A role for neural determination genes in specifying the dorsoventral identity of telencephalic neurons. Genes Dev. 14, 67-80.

Garcia-Moreno, F., Vasistha, N. A., Begbie, J. and Molnar, Z. (2014). CLoNe is a new method to target single progenitors and study their progeny in mouse and chick. Development 141, 1589-1598.

Gowan, K., Helms, A. W., Hunsaker, T. L., Collisson, T., Ebert, P. J., Odom, R. and Johnson, J. E. (2001). Crossinhibitory Activities of Ngn1 and Math1 Allow Specification of Distinct Dorsal Interneurons. Neuron 31, 219-232.

Guo, T., Liu, G., Du, H., Wen, Y., Wei, S., Li, Z., Tao, G., Shang, Z., Song, X., Zhang, Z., et al. (2019). Dlx1/2 are Central and Essential Components in the
Transcriptional Code for Generating Olfactory Bulb Interneurons. Cereb. Cortex 29, 4831-4849.

Han, S., Wilkinson, G. A., Okawa, S., Adnani, L., Dixit, R., Faisal, I., Brooks, M., Cortay, V., Chinchalongporn, V., Zinyk, D., et al. (2020). Proneural genes define ground state rules to regulate neurogenic patterning and cortical folding. Developmental Biology.

Hevner, R. F. (2006). From Radial Glia to PyramidalProjection Neuron: Transcription Factor Cascades in Cerebral Cortex Development. Mol. Neurobiol. 33, 033050.

Huang, W., Bhaduri, A., Velmeshev, D., Wang, S., Wang, L., Rottkamp, C. A., Alvarez-Buylla, A., Rowitch, D. H. and Kriegstein, A. R. (2020). Origins and Proliferative States of Human Oligodendrocyte Precursor Cells. Cell 182, 594-608.e11.

Jang, E. S. and Goldman, J. E. (2011). Pax6 Expression Is Sufficient to Induce a Neurogenic Fate in Glial Progenitors of the Neonatal Subventricular Zone. PLoS ONE 6, e20894.

Jiang, Q., Zagozewski, J., Godbout, R. and Eisenstat, D. D. (2020). Distal-less genes Dlx1/Dlx2 repress oligodendrocyte genesis through transcriptional inhibition of Olig2 expression in the developing vertebrate forebrain. Developmental Biology.

Johnson, W. E., Li, C. and Rabinovic, A. (2007). Adjusting batch effects in microarray expression data using empirical Bayes methods. Biostatistics 8, 118-127.

Kelenis, D. P., Hart, E., Edwards-Fligner, M., Johnson, J. E. and Vue, T. Y. (2018). ASCL1 regulates proliferation of NG2-glia in the embryonic and adult spinal cord. Glia $66,1862-1880$.

Kim, E. J., Battiste, J., Nakagawa, Y. and Johnson, J. E. (2008). Ascl1 (Mash1) lineage cells contribute to discrete cell populations in CNS architecture. Mol. Cell. Neurosci. 38, 595-606.

Kim, E. J., Ables, J. L., Dickel, L. K., Eisch, A. J. and Johnson, J. E. (2011). Ascl1 (Mash1) Defines Cells with Long-Term Neurogenic Potential in Subgranular and Subventricular Zones in Adult Mouse Brain. PLoS ONE 6, e18472.

Kohwi, M., Osumi, N., Rubenstein, J. L. R. and AlvarezBuylla, A. (2005). Pax6 Is Required for Making Specific Subpopulations of Granule and Periglomerular Neurons in the Olfactory Bulb. J. Neurosci. 25, 69977003.

Kovach, C., Dixit, R., Li, S., Mattar, P., Wilkinson, G., Elsen, G. E., Kurrasch, D. M., Hevner, R. F. and Schuurmans, C. (2013). Neurog2 Simultaneously Activates and Represses Alternative Gene Expression Programs in the Developing Neocortex. Cereb. Cortex 23, 1884-1900.

Kowalczyk, T., Pontious, A., Englund, C., Daza, R. A. M., Bedogni, F., Hodge, R., Attardo, A., Bell, C., Huttner, 
W. B. and Hevner, R. F. (2009). Intermediate Neuronal Progenitors (Basal Progenitors) Produce PyramidalProjection Neurons for All Layers of Cerebral Cortex. Cereb. Cortex 19, 2439-2450.

Kroll, T. T. and O'Leary, D. D. M. (2005). Ventralized dorsal telencephalic progenitors in Pax6 mutant mice generate GABA interneurons of a lateral ganglionic eminence fate. Proc. Natl. Acad. Sci. 102, 7374-7379.

La Manno, G., Soldatov, R., Zeisel, A., Braun, E., Hochgerner, H., Petukhov, V., Lidschreiber, K., Kastriti, M. E., Lönnerberg, P., Furlan, A., et al. (2018). RNA velocity of single cells. Nature 560, 494-498.

La Manno, G., Siletti, K., Furlan, A., Gyllborg, D., Vinsland, E., Langseth, C. M., Khven, I., Johnsson, A., Nilsson, M., Lönnerberg, P., et al. (2020). Molecular architecture of the developing mouse brain. Developmental Biology.

Li, J., Wang, C., Zhang, Z., Wen, Y., An, L., Liang, Q., Xu, Z., Wei, S., Li, W., Guo, T., et al. (2018). Transcription Factors Sp8 and Sp9 Coordinately Regulate Olfactory Bulb Interneuron Development. Cereb. Cortex 28, 3278-3294.

Lim, Y., Cho, I.-T., Shi, X., Grinspan, J. B., Cho, G. and Golden, J. A. (2019). Arx Expression Suppresses Ventralization of the Developing Dorsal Forebrain. Sci. Rep. 9, 226.

Lindtner, S., Catta-Preta, R., Tian, H., Su-Feher, L., Price, J. D., Dickel, D. E., Greiner, V., Silberberg, S. N., McKinsey, G. L., McManus, M. T., et al. (2019). Genomic Resolution of DLX-Orchestrated Transcriptional Circuits Driving Development of Forebrain GABAergic Neurons. Cell Rep. 28, 20482063.e8.

Macosko, E. Z., Basu, A., Satija, R., Nemesh, J., Shekhar, K., Goldman, M., Tirosh, I., Bialas, A. R., Kamitaki, N., Martersteck, E. M., et al. (2015). Highly Parallel Genome-wide Expression Profiling of Individual Cells Using Nanoliter Droplets. Cell 161, 1202-1214.

Madisen, L., Zwingman, T. A., Sunkin, S. M., Oh, S. W., Zariwala, H. A., Gu, H., Ng, L. L., Palmiter, R. D., Hawrylycz, M. J., Jones, A. R., et al. (2010). A robust and high-throughput Cre reporting and characterization system for the whole mouse brain. Nat. Neurosci. 13, 133-140.

Marisca, R., Hoche, T., Agirre, E., Hoodless, L. J., Barkey, W., Auer, F., Castelo-Branco, G. and Czopka, T. (2020). Functionally distinct subgroups of oligodendrocyte precursor cells integrate neural activity and execute myelin formation. Nat. Neurosci. 23, 363374.

Marques, S., Zeisel, A., Codeluppi, S., van Bruggen, D., Mendanha Falcao, A., Xiao, L., Li, H., Haring, M., Hochgerner, H., Romanov, R. A., et al. (2016). Oligodendrocyte heterogeneity in the mouse juvenile and adult central nervous system. Science 352, 13261329.

Nakatani, H., Martin, E., Hassani, H., Clavairoly, A., Maire, C. L., Viadieu, A., Kerninon, C., Delmasure, A., Frah, M., Weber, M., et al. (2013). Ascl1/Mash1 Promotes Brain Oligodendrogenesis during Myelination and Remyelination. J. Neurosci. 33, 97529768.

Parras, C. M., Galli, R., Britz, O., Soares, S., Galichet, C., Battiste, J., Johnson, J. E., Nakafuku, M., Vescovi, A. and Guillemot, F. (2004). Mash1 specifies neurons and oligodendrocytes in the postnatal brain. $E M B O \mathrm{~J} . \mathbf{2 3}$, 4495-4505.

Parras, C. M., Hunt, C., Sugimori, M., Nakafuku, M., Rowitch, D. and Guillemot, F. (2007). The Proneural Gene Mash1 Specifies an Early Population of Telencephalic Oligodendrocytes. J. Neurosci. 27, 42334242.

Petryniak, M. A., Potter, G. B., Rowitch, D. H. and Rubenstein, J. L. R. (2007). Dlx1 and Dlx2 Control Neuronal versus Oligodendroglial Cell Fate Acquisition in the Developing Forebrain. Neuron 55, 417-433.

Pollen, A. A., Nowakowski, T. J., Chen, J., Retallack, H., Sandoval-Espinosa, C., Nicholas, C. R., Shuga, J., Liu, S. J., Oldham, M. C., Diaz, A., et al. (2015). Molecular Identity of Human Outer Radial Glia during Cortical Development. Cell 163, 55-67.

Roybon, L., Mastracci, T. L., Ribeiro, D., Sussel, L., Brundin, P. and Li, J.-Y. (2010). GABAergic Differentiation Induced by Mash1 Is Compromised by the bHLH Proteins Neurogenin2, NeuroD1, and NeuroD2. Cereb. Cortex 20, 1234-1244.

Scardigli, R. (2003). Direct and concentration-dependent regulation of the proneural gene Neurogenin2 by Pax6. Development 130, 3269-3281.

Schuurmans, C., Armant, O., Nieto, M., Stenman, J. M., Britz, O., Klenin, N., Brown, C., Langevin, L.-M., Seibt, J., Tang, H., et al. (2004). Sequential phases of cortical specification involve Neurogenin-dependent and independent pathways. EMBO J. 23, 2892-2902.

Silbereis, J. C., Nobuta, H., Tsai, H.-H., Heine, V. M., McKinsey, G. L., Meijer, D. H., Howard, M. A., Petryniak, M. A., Potter, G. B., Alberta, J. A., et al. (2014). Olig1 Function Is Required to Repress Dlx1/2 and Interneuron Production in Mammalian Brain. Neuron 81, 574-587.

Street, K., Risso, D., Fletcher, R. B., Das, D., Ngai, J., Yosef, N., Purdom, E. and Dudoit, S. (2018). Slingshot: cell lineage and pseudotime inference for single-cell transcriptomics. BMC Genomics 19, 477.

Sugimori, M., Nagao, M., Bertrand, N., Parras, C. M., Guillemot, F. and Nakafuku, M. (2007). Combinatorial actions of patterning and HLH transcription factors in the spatiotemporal control of neurogenesis and 
gliogenesis in the developing spinal cord. Development $134,1617-1629$.

Sugimori, M., Nagao, M., Parras, C. M., Nakatani, H., Lebel, M., Guillemot, F. and Nakafuku, M. (2008). Ascl1 is required for oligodendrocyte development in the spinal cord. Development 135, 1271-1281.

Sun, J., Rockowitz, S., Xie, Q., Ashery-Padan, R., Zheng, D. and Cvekl, A. (2015). Identification of in vivo DNAbinding mechanisms of Pax6 and reconstruction of Pax6-dependent gene regulatory networks during forebrain and lens development. Nucleic Acids Res. 43, 6827-6846.

Toresson, H., Potter, S. S. and Campbell, K. (2000). Genetic control of dorsal-ventral identity in the telencephalon: opposing roles for Pax6 and Gsh2. Dev. Camb. Engl. 127, 4361-4371.

Van den Berge, K., Roux de Bézieux, H., Street, K., Saelens, W., Cannoodt, R., Saeys, Y., Dudoit, S. and Clement, L. (2020). Trajectory-based differential expression analysis for single-cell sequencing data. Nat. Commun. 11, 1201.

Voronova, A., Fischer, A., Ryan, T., Al Madhoun, A. and Skerjanc, I. S. (2011). Ascl1/Mash1 is a novel target of Gli2 during Gli2-induced neurogenesis in P19 EC cells. Plos One 6, e19174.

Vue, T. Y., Kim, E. J., Parras, C. M., Guillemot, F. and Johnson, J. E. (2014). Ascl1 controls the number and distribution of astrocytes and oligodendrocytes in the gray matter and white matter of the spinal cord. Dev. Camb. Engl. 141, 3721-3731.

Vue, T. Y., Kollipara, R. K., Borromeo, M. D., Smith, T., Mashimo, T., Burns, D. K., Bachoo, R. M. and Johnson, J. E. (2020). ASCL1 regulates neurodevelopmental transcription factors and cell cycle genes in brain tumors of glioma mouse models. Glia glia.23873.

Waclaw, R. R., Wang, B., Pei, Z., Ehrman, L. A. and Campbell, K. (2009). Distinct Temporal Requirements for the Homeobox Gene Gsx2 in Specifying Striatal and Olfactory Bulb Neuronal Fates. Neuron 63, 451-465.

Wang, L., Zhang, Z. G., Gregg, S. R., Zhang, R. L., Jiao, Z., LeTourneau, Y., Liu, X., Feng, Y., Gerwien, J., Torup, L., et al. (2007). The Sonic hedgehog pathway mediates carbamylated erythropoietin-enhanced proliferation and differentiation of adult neural progenitor cells. $J$. Biol. Chem. 282, 32462-32470.

Wang, B., Waclaw, R. R., Allen, Z. J., Guillemot, F. and Campbell, K. (2009). Ascl1 is a required downstream effector of Gsx gene function in the embryonic mouse telencephalon. Neural Develop. 4, 5.

Winkler, C. C. and Franco, S. J. (2019). Loss of Shh signaling in the neocortex reveals heterogeneous cell recovery responses from distinct oligodendrocyte populations. Dev. Biol. 452, 55-65.

Winkler, C. C., Yabut, O. R., Fregoso, S. P., Gomez, H. G., Dwyer, B. E., Pleasure, S. J. and Franco, S. J. (2018). The Dorsal Wave of Neocortical Oligodendrogenesis Begins Embryonically and Requires Multiple Sources of Sonic Hedgehog. J. Neurosci. 38, 5237-5250.

Wolf, F. A., Angerer, P. and Theis, F. J. (2018). SCANPY: large-scale single-cell gene expression data analysis. Genome Biol. 19, 15.

Yabut, O. R. and Pleasure, S. J. (2018). Sonic Hedgehog Signaling Rises to the Surface: Emerging Roles in Neocortical Development. Brain Plast. 3, 119-128.

Yabut, O. R., Fernandez, G., Huynh, T., Yoon, K. and Pleasure, S. J. (2015). Suppressor of Fused Is Critical for Maintenance of Neuronal Progenitor Identity during Corticogenesis. Cell Rep. 12, 2021-2034.

Yabut, O., Ng, H., Fernandez, G., Yoon, K., Kuhn, J. and Pleasure, S. (2016). Loss of Suppressor of Fused in MidCorticogenesis Leads to the Expansion of Intermediate Progenitors. J. Dev. Biol. 4, 29.

Yabut, O. R., Ng, H.-X., Yoon, K., Arela, J. C., Ngo, T. and Pleasure, S. J. (2020). The Neocortical Progenitor Specification Program Is Established through Combined Modulation of SHH and FGF Signaling. J. Neurosci. 40, 6872-6887.

Yun, K., Potter, S. and Rubenstein, J. L. (2001). Gsh2 and Pax6 play complementary roles in dorsoventral patterning of the mammalian telencephalon. Dev. Camb. Engl. 128, 193-205.

Yung, S.-Y., Gokhan, S., Jurcsak, J., Molero, A. E., Abrajano, J. J. and Mehler, M. F. (2002). Differential modulation of BMP signaling promotes the elaboration of cerebral cortical GABAergic neurons or oligodendrocytes from a common sonic hedgehogresponsive ventral forebrain progenitor species. Proc. Natl. Acad. Sci. U. S. A. 99, 16273-16278.

Yuzwa, S. A., Borrett, M. J., Innes, B. T., Voronova, A., Ketela, T., Kaplan, D. R., Bader, G. D. and Miller, F. D. (2017). Developmental Emergence of Adult Neural Stem Cells as Revealed by Single-Cell Transcriptional Profiling. Cell Rep. 21, 3970-3986.

Zhang, Y., Liu, G., Guo, T., Liang, X. G., Du, H., Yang, L., Bhaduri, A., Li, X., Xu, Z., Zhang, Z., et al. (2020). Cortical Neural Stem Cell Lineage Progression Is Regulated by Extrinsic Signaling Molecule Sonic Hedgehog. Cell Rep. 30, 4490-4504.e4. 